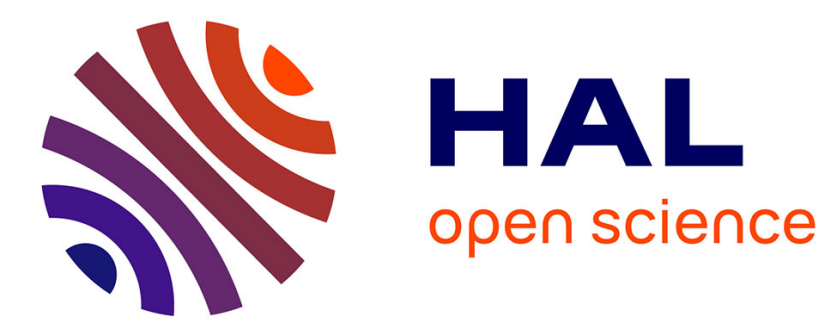

\title{
Long-time dynamics of coherent states in strong magnetic fields
}

\author{
Grégory Boil, San Vu Ngoc
}

\section{To cite this version:}

Grégory Boil, San Vu Ngoc. Long-time dynamics of coherent states in strong magnetic fields. American Journal of Mathematics, 2021, 143 (6), pp.1747-1789. 10.1353/ajm.2021.0045 . hal-01812732

\section{HAL Id: hal-01812732 \\ https://hal.science/hal-01812732}

Submitted on 11 Jun 2018

HAL is a multi-disciplinary open access archive for the deposit and dissemination of scientific research documents, whether they are published or not. The documents may come from teaching and research institutions in France or abroad, or from public or private research centers.
L'archive ouverte pluridisciplinaire HAL, est destinée au dépôt et à la diffusion de documents scientifiques de niveau recherche, publiés ou non, émanant des établissements d'enseignement et de recherche français ou étrangers, des laboratoires publics ou privés. 


\title{
LONG-TIME DYNAMICS OF COHERENT STATES IN STRONG MAGNETIC FIELDS
}

\author{
GRÉGORY BOIL, SAN VŨ NGỌC
}

\begin{abstract}
We consider a charged particle on a plane, subject to a strong, purely magnetic external field. It is well known that quantum dynamics closely follows the classical one for short periods of time, while for times larger than $\ln \frac{1}{\hbar}$, where $\hbar$ is Planck's constant, purely quantum phenomena are expected to happen. In this paper we follow the Schrödinger evolution of generalized coherent states for times of order $1 / \hbar$. We prove that, when the initial energy is low, the initial states splits into multiple wavepackets, each one following the average dynamics of the guiding center motion but at its own speed.
\end{abstract}

\section{INTRODUCTION}

1.1. Motivation. The aim of this article is to study the propagation of coherent states in the 2-dimensional plane $\mathbb{R}^{2} \subset \mathbb{R}^{3}$, subject to a strong magnetic field $\vec{B}=B e_{3}$ perpendicular to the plane. In this article, $B$ will be a $C^{\infty}$ smooth, non vanishing function $B: \mathbb{R}^{2} \rightarrow(0,+\infty)$. From the Poincaré lemma, one can find a smooth function $A: \mathbb{R}^{2} \rightarrow \mathbb{R}^{2}$, the magnetic potential, such that

$$
\mathrm{d} A:=\partial_{2} A_{1}-\partial_{1} A_{2}=B .
$$

The classical magnetic Hamiltonian is

$$
H: \mathbb{R}^{2} \times \mathbb{R}^{2} \ni(q, p) \mapsto\|p-A(q)\|^{2} \in \mathbb{R},
$$

giving rise to the Hamiltonian flow $\Phi_{H}^{t}\left(q_{0}, p_{0}\right)=(q(t), p(t))$ defined by Hamilton's equations

$$
\left\{\begin{array}{l}
\dot{q}(t)=\frac{\partial H}{\partial p}(q(t), p(t)) \\
\dot{p}(t)=-\frac{\partial H}{\partial q}(q(t), p(t)),
\end{array}\right.
$$

with initial condition $q(0)=q_{0}, p(0)=p_{0}$. The corresponding quantum operator, or magnetic Laplacian, is the differential operator given by

$$
\mathcal{L}_{\hbar, A}:=\|-i \hbar \nabla-A(q)\|^{2}=\left(-i \hbar \partial_{1}-A_{1}(q)\right)^{2}+\left(-i \hbar \partial_{2}-A_{2}(q)\right)^{2} .
$$

Date: June 10, 2018. 
It is well known that $\mathcal{L}_{\hbar, A}$, as an unbounded operator acting on $L^{2}\left(\mathbb{R}^{2}\right)$, is essentially self-adjoint [18], and one can define the associated Schrödinger unitary group on $L^{2}\left(\mathbb{R}^{2}\right)$ given by

$$
\left\{\begin{array}{l}
i \hbar \partial_{t} \varphi_{\hbar}^{t}=\mathcal{L}_{\hbar, A} \varphi_{\hbar}^{t} \\
\varphi_{\hbar}^{t=0}=\varphi_{\hbar}^{0}
\end{array}\right.
$$

We shall consider the case where the initial quantum state $\varphi_{\hbar}^{0}$ is coherent, which means strongly localized in phase space (see Section 2). The general semiclassical theory states that, for finite times (by finite, we mean a time $t$ that does not depend on $\hbar$ ), the quantum evolution (1.3) closely follows the classical trajectories (1.1), in the regime where $\hbar$ is small. Early works in semiclassical physics and chemistry emphasize the importance of this idea, see [20, 17]. More precisely, one can define the classical limit (or semiclassical wavefront set, see Definition 2.16) of a coherent state; this is a position $z=(q, p)$ in phase space. Then, if $z_{0}$ denotes the classical limit of the initial quantum state $\varphi_{\hbar}^{0}$, Egorov's theorem (Proposition 2.9) ensures that the classical limit of $\varphi_{\hbar}^{t}$ coincides with the flow at time $t$ of the classical Hamiltonian starting from $z_{0}$. See for instance [11] for a mathematical account of this in the case of electric potentials, or $[27,32]$ for a more general formulation.

However, for long times, i.e. times that tend to infinity when $\hbar \rightarrow 0$, most of the results break down. In many cases, the non-commutativity of the limits $\hbar \rightarrow 0$ and $t \rightarrow \infty$ is simply inextricable, at least beyond the so-called Ehrenfest time $t \asymp|\ln \hbar|$. In this paper, we will investigate the propagation of a coherent quantum wave packet under the action of strong magnetic fields, for times of order $t \asymp 1 / \hbar$. How is this possible?

Classical trajectories of a charged particle under a strong magnetic field have a distinctive feature: they can be approximately described as a superposition of a fast rotation motion (cyclotron, or Larmor motion) with a slow drifting motion (often called the guiding center motion [20]), see Figure 1. If the energy of the initial state is small enough, then this guiding center motion can be controlled, and the fast rotation is almost decoupled, giving rise to an adiabatic invariant (see the classical book [21], or the recent review [3]). Then, since we work on a two-dimensional plane, the motion becomes nearly integrable. This near-integrability is a crucial element in an attempt to explain why it is possible to describe the motion for such long times.

1.2. Mathematical background. We shall assume that the magnetic field $B$ and its derivatives have at most polynomial growth at infinity, in the sense that $B$ belongs to a symbol class $S(m)$ for some order function $m$ on $\mathbb{R}^{2}$ (see Section 2.2). For instance, $m(q)=\left(\|q\|^{2}+1\right)^{M}$ for some $M \in \mathbb{R}$. Then one can find a potential $A$ lying in some $S\left(m^{\prime}\right)$, with an order function $m^{\prime}$ on $\mathbb{R}^{2}$, showing that $H \in S\left(m^{\prime \prime}\right)$ for an order function $m^{\prime \prime}$ on $\mathbb{R}^{4}$. The magnetic Laplacian defined in (1.2) is the natural 


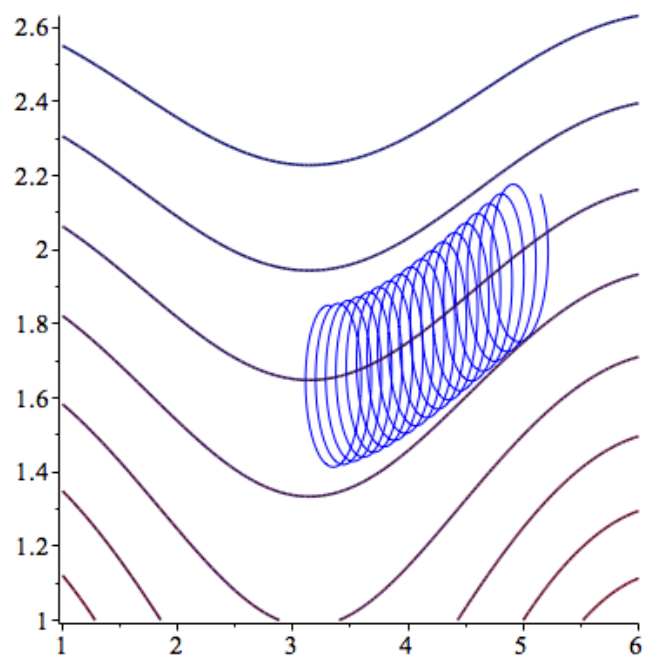

Figure 1. Motion of a classical particle in a strong magnetic field. The particle oscillates around a level line of $B$. Here $B\left(q_{1}, q_{2}\right)=2-\cos \left(q_{1}\right)+q_{2}^{2}$.

symmetric (Weyl) quantization of $H$, acting of the Schwartz space $\mathscr{S}\left(\mathbb{R}^{2}\right)$ :

$$
\mathcal{L}_{\hbar, A}:=\mathrm{Op}_{\hbar}^{w}(H) .
$$

Since $\mathcal{L}_{\hbar, A}$ is essentially self-adjoint on $L^{2}\left(\mathbb{R}^{2}\right)$ we will identify $\mathcal{L}_{\hbar, A}$ with its selfadjoint extension, and we may now consider the magnetic Schrödinger evolution equation (1.3), where $\varphi_{\hbar}^{0} \in \mathscr{S}\left(\mathbb{R}^{2}\right)$. From Stone's theorem (see e.g. [26]), there is a unique continuous family of unitary operators $\left(P^{t}\right)_{t \in \mathbb{R}}$ satisfying the propagation equation

$$
\left\{\begin{array}{l}
i \hbar \partial_{t} P^{t}=\mathcal{L}_{\hbar, A} P^{t} \\
P^{t=0}=i d_{\mathrm{L}^{2}\left(\mathbb{R}^{2}\right)}
\end{array}\right.
$$

and for any $t \in \mathbb{R}$, the solution to (1.3) is given by $\varphi_{\hbar}^{t}:=P^{t} \varphi_{\hbar}^{0}$. In this work, $\varphi_{\hbar}^{0}$ will be a coherent state in a generalized sense, as follows (see Section 2 for a precise definition).

Defining for $\hbar>0$ and $z=(q, p) \in \mathbb{R}^{2} \times \mathbb{R}^{2}$ the rescaled translation operator

$$
T_{\hbar}(z) \circ \Lambda_{\hbar}: \mathrm{L}^{2}\left(\mathbb{R}^{2}\right) \ni f \mapsto\left[x \mapsto \frac{1}{\sqrt{\hbar}} e^{-\frac{i}{2 \hbar} q \cdot p} e^{\frac{i}{\hbar} x \cdot p} f\left(\frac{x-q}{\sqrt{\hbar}}\right)\right]
$$

a state $\varphi_{z}$ will be called a coherent state if one can find a function $f \in \mathscr{S}\left(\mathbb{R}^{2}\right)$ with normalized $\mathrm{L}^{2}$-norm, a family of functions $\left(g_{\hbar}\right)_{\hbar \in\left(0, \hbar_{0}\right)} \in \mathscr{S}\left(\mathbb{R}^{2}\right)$ with seminorms that are uniformly bounded in $\hbar$, and a real number $\beta>0$, such that

$$
\varphi_{z}:=T_{\hbar}(z) \Lambda_{\hbar} \cdot\left(f+\hbar^{\beta} g_{\hbar}\right) .
$$


Such a coherent state is said to be centered at $z$ in phase space. We denote by $\mathfrak{C}$ the set of coherent states. Since we are interested in the propagation of quantum states with low energy, we shall consider coherent states centered at a vanishing point of the Hamiltonian $H$, i.e. $z \in \Sigma$ with

$$
\Sigma:=H^{-1}(\{0\})=\left\{(q, A(q)) \mid q \in \mathbb{R}^{2}\right\} \subset \mathbb{R}_{q}^{2} \times \mathbb{R}_{p}^{2} .
$$

On this smooth surface we define the pull-back $\tilde{B}$ of the magnetic field $B$ as follows. Let $j$ be the projection

$$
j: \Sigma \ni(q, A(q)) \mapsto q \in \mathbb{R}_{q}^{2} .
$$

Since $\Sigma$ is the graph of the function $A, j$ is invertible and then we can view the magnetic field as a function on $\Sigma$ via

$$
\tilde{B}:=B \circ j
$$

One can check that, when $B$ is non-vanishing, $\Sigma$ is a symplectic submanifold of the canonical phase space $\mathbb{R}^{2} \times \mathbb{R}^{2}=T^{*} \mathbb{R}^{2}$ (in fact, the restriction to $\Sigma$ of the canonical symplectic form on $T^{*} \mathbb{R}^{2}$ is exactly the pull-back $\left.j^{*}\left(B d q_{1} \wedge d q_{2}\right)\right)$. Hence $\tilde{B}$ is now a Hamiltonian on $\Sigma$ and we can consider its Hamiltonian flow $\Phi_{\tilde{B}}^{t}$. A basic result in magnetic dynamics (see also [25]) is that this flow gives a good approximation of the guiding center motion.

1.3. Description of the main results. The main goal is to prove the following fact. Let $\varphi_{\hbar}^{0}$ be a coherent state centered at a point $z$ in the characteristic surface $\Sigma$. Classically, this state cannot move because it has zero kinetic energy. However, due to the uncertainty principle, the quantum state lives on a ball of radius $\asymp \hbar^{1 / 2}$ around $z$, and so almost all the points in this ball will actually move slowly, at various speeds of order $\hbar$, with a precise factor depending on the distance to $\Sigma$. But, because of energy quantization, the involved speeds will actually be quantized: only integer multiples of $\hbar B_{0}$, for some positive constant $B_{0}$, will play a part. Thus, the initial state will be split into a number of new coherent states evolving at theses speed scales, becoming genuinely separated from each other after a time of order $\asymp \hbar^{-1}$.

Theorem 1.1. There exists a self-adjoint pseudodifferential operator $\mathcal{J}_{\hbar}$ (the 'Quantum adiabatic invariant') on $\mathrm{L}^{2}\left(\mathbb{R}^{2}\right)$ such that for any $\alpha \in(0,1)$ and $K>0$, if $J_{\hbar} \in \mathbb{N}$ is a family of integers satisfying $J_{\hbar} \geqslant K \hbar^{-\alpha}$, then the following holds.

Let $\varphi_{\hbar}^{0} \in \mathfrak{C}$ be a coherent state centered in $z_{0} \in \Sigma=H^{-1}(0)$ and consider its propagation $\left(\varphi_{\hbar}^{t}\right)_{t \in \mathbb{R}}$ through the Schrödinger equation (1.3):

$$
\forall t \in \mathbb{R}, \varphi_{\hbar}^{t}:=P^{t} \varphi_{\hbar}^{0}
$$


Then $\varphi_{\hbar}^{t}$ can be decomposed as follows:

$$
\varphi_{\hbar}^{t}=\sum_{j=0}^{J_{\hbar}} \alpha_{j} \varphi_{j, \hbar}^{t}+r_{\hbar}^{t}
$$

where $\left(\alpha_{j}\right)_{j \in \mathbb{N}} \in \ell^{2}\left(\mathbb{R}_{+}\right)$, there exists $T>0$ such that the remainder $r_{\hbar}^{t}$ satisfies, for any $N \in \mathbb{N}$,

$$
\sup _{t \in[0, T / \hbar]}\left\|\left(\mathcal{L}_{\hbar, A}\right)^{N} r_{\hbar}^{t}\right\|_{L^{2}\left(\mathbb{R}^{2}\right)}=\mathcal{O}\left(\hbar^{\infty}\right),
$$

and the $\varphi_{j, \hbar}^{t}$ have the following properties.

(1) $\forall j \geqslant 0, \alpha_{j} \varphi_{j, \hbar}^{t}$ is the orthogonal projection of $\varphi_{\hbar}^{t}$ onto $\operatorname{ker}\left(\mathcal{J}_{\hbar}-(2 j+1) \hbar\right)$;

(2) $\forall \tilde{t} \in[0, T]$,

$$
\varphi_{j, \hbar}^{\tilde{t} / \hbar} \in \mathfrak{C}
$$

(3) we can follow the center of these coherent states : for any $\tilde{t} \in[0, T]$, we have

$$
\mathcal{W F}\left(\varphi_{j, \hbar}^{\tilde{t} / \hbar}\right)=\{z((2 j+1) \tilde{t})\}
$$

where $z(\tilde{t}):=\Phi_{\tilde{B}}^{\tilde{t}} z_{0}$, and $\Phi_{\tilde{B}}^{\tilde{t}}$ is the Hamiltonian flow of $\tilde{B}$ on $\Sigma$.

Here, $\mathcal{W F}(f)$ denotes the semiclassical wavefront set of the function $f \in \mathscr{S}\left(\mathbb{R}^{2}\right)$, see Definition 2.16 below. The quantum adiabatic invariant $\mathcal{J}_{\hbar}$ corresponds to the pure cyclotron motion that one would obtain for a homogeneous magnetic field of strength 1. Its spectrum consists of the eigenvalues $\{(2 j+1) \hbar ; j \in \mathbb{N}\}$, and the corresponding eigenspaces

$$
\mathcal{H}_{j, \hbar}=\operatorname{ker}\left(\mathcal{J}_{\hbar}-(2 j+1) \hbar\right)
$$

are infinite dimensional Hilbert spaces that one can call the adiabatic Landau levels of the system. Up to an error of size $\mathcal{O}\left(\hbar^{\infty}\right)$, these spaces are preserved by the quantum dynamics. We see from (1.7) that the "adiabatic value" $(2 j+1) \hbar$ is coupled with the original dynamics in that it defines a quantized speed of motion for a coherent state living in $\mathcal{H}_{j, \hbar}$. Since, to the best of our knownledge, the description given by Theorem 1.1 is new, it would be very interesting to observe this quantization effect in a (real or numerical) experiment; but the long time $\hbar^{-1}$ clearly poses serious numerical challenges.

Notice that the remainder in (1.5) is controlled in terms of the obvious Sobolev-like norms that are preserved by the dynamics: the $\mathrm{L}^{2}$-norms of arbitrary powers of the magnetic Laplacian (which, in particular, control the quadratic form $\left\langle\mathcal{L}_{\hbar, A} \psi, \psi\right\rangle$, and hence, by the Cauchy-Schwarz inequality, the norm of the usual magnetic Sobolev space $H_{A}^{1}$, where derivatives are replaced by magnetic derivatives $\hbar \partial_{j}+A_{j}$ ). But recall that $\mathcal{L}_{\hbar, A}$ is not elliptic in the semiclassical sense, since the characteristic manifold 
$\Sigma$ extends to infinity in $q$. Therefore, if one is interested in quantum transport or, rather, quantum localization, it is in general an important, non obvious question to check whether, and to which extent, these norms can be compared to more 'localized' norms like Schwartz seminorms or standard Sobolev norms. The answer is expected to depend on the geometry of the magnetic field. Recently, the control of Schwartz seminorms has been obtained in [2], under the assumption that the magnetic field is uniformly non-vanishing, and satisfies the following property.

$$
\begin{aligned}
& \text { (P) : for all } \alpha \in \mathbb{N}^{2} \text {, there exists } C>0 \text { such that, for all } x \in \mathbb{R}^{2}, \\
& \qquad \partial^{\alpha} B(x)\|\leqslant C\| B(x) \| .
\end{aligned}
$$

This assumption states that the derivatives of the magnetic field are controlled by the magnetic field itself, preventing $B$ from 'oscillating too much' at infinity. It appears to be rather known and used in literature. In dimension $d \geqslant 3$, this property is crucial to obtain that, under some ellipticity condition, $\mathcal{L}_{\hbar, A}$ has compact resolvent and hence no essential spectrum; and if these ellipticity conditions do not hold, there is a rather precise description of the essential spectrum (see $[1,15])$. The lower bound at the bottom of the essential spectrum of $\mathcal{L}_{\hbar, A}$ obtained in [16] is an important ingredient in the localization estimates of [2].

In general, Property $(\mathbf{P})$ is optimal to prove the compactness of the resolvent: in the paper [8], the author constructs a magnetic field that does not satisfy this property and such that $\mathcal{L}_{\hbar, A}$, while enjoying some ellipticity properties, has no compact resolvent. On the other hand, in dimension 2, the diamagnetic inequality gives an immediate lower bound on the essential spectrum without needing $(\mathbf{P})$. It would be interesting to investigate whether, in dimension 2, the localization estimates of [2] could be obtained under a weaker condition than $(\mathbf{P})$.

In this work, we take advantage of the results of [2] to obtain a stronger estimate of the remainder $r_{\hbar}^{t}$ in Theorem 1.1, as follows.

Theorem 1.2. With the same notations as in Theorem 1.1, for a magnetic field $B$ satisfying the above property $(\boldsymbol{P})$, assuming that there exists $b_{0}>0$ such that

$$
\forall q \in \mathbb{R}^{2}, B(q) \geqslant b_{0},
$$

we get the following estimate of $r_{\hbar}^{t}:$ given any $T \in \mathbb{R}$ and any Schwartz seminorm $p$, we have

$$
\sup _{t \in[0, T / \hbar]} p\left(r_{\hbar}^{t}\right)=\mathcal{O}\left(\hbar^{\infty}\right)
$$

We surmise that this result does not hold for general magnetic fields $B$. Although the control in terms of Schwartz seminorms is much more satisfying in the setting of coherent states, the price to pay for this is that the proof of Theorem 1.2 is substantially more involved than the proof of Theorem 1.1. 
1.4. Organization of the article. In section 2, we define the class of coherent states that we will use, and we present some facts about their propagation. We will prove some short time propagation properties for these coherent states and some long-time estimates in the case of coherent states centered at a critical point of the classical dynamics. In section 3, we introduce the symplectic and quantum magnetic normal forms, which are here the main tools to study the long time dynamics. In section 4, we prove Theorems 1.1 and 1.2.

\section{A general Class of COHERENT STATES AND RELATED PROPAGATION}

\section{RESULTS}

The goal of this section is to introduce a class of 'coherent states', which is large enough to contain the usual ones (such as the Gaussian coherent states and the squeezed ones - see for instance [4]), but also more flexible, allowing to replace exponential localization by a softer Schwartz-like 'rapid decay'. As the following discussion holds in any dimension, in this paper we will not limit ourselves to the dimension 2, but we will consider instead the general dimension $n$. Recall that the Schwartz space $\mathscr{S}\left(\mathbb{R}^{n}\right)$ has the Fréchet topology induced by the seminorms

$$
p_{m, N}(f):=\sup _{\substack{x \in \mathbb{R}^{n} \\ \alpha \in \mathbb{N}^{n},\left|\leqslant m \\ \beta \in \mathbb{N}^{n},\right| \beta \mid \leqslant N}}\left|x^{\beta} \partial^{\alpha} f(x)\right|, \quad m, N \in \mathbb{N} .
$$

2.1. Definition of the class. We consider first $\mathfrak{s}^{[n]}$, the set of shapes of coherent states defined as follows.

Definition 2.1. We say that $\mathrm{f}_{\hbar} \in \mathfrak{s}^{[n]}$ if and only if one can find $f \in \mathscr{S}\left(\mathbb{R}^{n}\right)$ with $\|f\|_{\mathrm{L}^{2}\left(\mathbb{R}^{n}\right)}=1$, a family $\left(g_{\hbar}\right)_{\hbar \in\left(0, \hbar_{0}\right)} \subset \mathscr{S}\left(\mathbb{R}^{n}\right)$ with seminorms uniformly bounded with respect to $\hbar$, and a real number $\beta>0$ such that

$$
\mathrm{f}_{\hbar}=f+\hbar^{\beta} g_{\hbar}
$$

In the definition below, we use the following unitary operators on $\mathrm{L}^{2}\left(\mathbb{R}^{n}\right)$ : the rescaling operator

$$
\left(\Lambda_{\hbar} u\right)(x)=\frac{1}{\hbar^{n / 4}} u\left(\frac{x}{\sqrt{\hbar}}\right)
$$

and the translation operator

$$
\forall z=(q, p) \in \mathbb{R}^{2 n}, \quad\left(T_{\hbar}(z) u\right)(x)=e^{-\frac{i}{2 \hbar} q \cdot p} e^{\frac{i}{\hbar} x \cdot p} u(x-q) .
$$

Definition 2.2. A coherent state $\varphi_{\hbar}$ in the class $\mathfrak{C}^{[n]}$ is given by its center $z$ in the phase space $\mathbb{R}_{q}^{2} \times \mathbb{R}_{p}^{2}$, a phase $\delta \in \mathbb{R}$ and a shape $\mathrm{f}_{\hbar} \in \mathfrak{s}^{[n]}$ by the formula

$$
\varphi_{\hbar}=e^{-i \delta / \hbar} T_{\hbar}(z) \Lambda_{\hbar} \cdot \mathrm{f}_{\hbar} .
$$


In other words, we have

$$
\mathfrak{C}^{[n]}:=\left\{\varphi_{\hbar}=e^{-i \delta / \hbar} T_{\hbar}(z) \Lambda_{\hbar} \cdot \mathrm{f}_{\hbar} \quad \mid \quad \delta \in \mathbb{R}, z \in \mathbb{R}^{2 n}, \mathrm{f}_{\hbar} \in \mathfrak{s}^{[n]}\right\} .
$$

We will denote $\mathfrak{C}:=\mathfrak{C}^{[2]}$. In this text, unless explicitly stated, the expression 'coherent state' will always refer to an element of $\mathfrak{C}$. This definition generalizes the ones in [11] or [6] that only allow a $\hbar$ independent part in the shape $f_{\hbar}$. In [24, 22], the author uses a full expansion in $\hbar^{1 / 2}$ for the shape in $\mathscr{S}\left(\mathbb{R}^{n}\right)$ in the Borel sense. In this paper, the shape of the coherent state admits an expansion at the first order in any $\hbar^{\beta}$ in the shape, $\beta>0$, bounded in $\hbar$ in $\mathscr{S}\left(\mathbb{R}^{n}\right)$. Finally, an interesting thing in this definition is that, using results in [5, 6, 29], under short-time propagation this class must remain stable. This point will be dealt with in what follows.

2.2. Propagation results. A first and natural question is 'How is such a coherent state propagated for finite times?'. The propagation of coherent states has been widely studied over the last few decades. A lot of results are related to approximation of the propagation of coherent states by sums of coherent states whose parameters depend on time. In $[11,12,13]$ we find such propagation results for finite times and for Gaussian like coherent states, defined as the squeezed states in [6]. In [14, 10], the authors investigate the propagation for longer times, reaching Ehrenfest times, i.e. times of order $|\log (\hbar)|$ and the bounds in these approximations are precised in [5] in terms of the the linearized flow around the underlying classical dynamics, using the well-known propagation of purely Gaussian coherent states through quadratic Hamiltonians and the theory of metaplectic operators. One can find a precise review of the quadratic propagation of coherent states in $[28,22]$. In [31] the authors investigate expansion in power of $\hbar$ instead of $\hbar^{1 / 2}$ of the dynamics of coherent states defined in the formalism of Lagrangian states. Nevertheless, due to the analysis of the turning points, this study holds for short times in the propagation. Finally, we can state the work done in $[23,24]$ where the authors use coherent states and their propagation in order to build quasimodes for general Schrodinger operators

We now present some facts about the propagation of coherent states in the class $\mathfrak{C}^{[n]}$, and some Schwartz estimates of these propagations. Our goal is not to approximate the propagation by expansions of coherent states but, because of the general definition of the shape $\mathrm{f}_{\hbar}$, to turn these results into a stability property of the class through finite time propagation. We also give estimates on these propagations. To do so, it will be convenient to introduce the semiclassical Sobolev spaces, which are more suitable for pseudodifferential computations. We recall here their definition, and refer to [32, Chapter 8] for a more detailed presentation.

An order function on $\mathbb{R}^{2 n}$ is a function $m: \mathbb{R}^{2 n} \rightarrow \mathbb{R}$ for which there is an integer $N$ and a constant $C>0$ such that for any $X, Y \in \mathbb{R}^{2 n}$

$$
m(X) \leqslant C\langle X-Y\rangle^{N} m(Y)
$$


where $\langle X\rangle:=\left(1+\|X\|^{2}\right)^{1 / 2},\|\cdot\|$ denoting the euclidian norm on $\mathbb{R}^{n}$ (see for instance [7, Definition 7.4]). The symbol class associated with this function is

$$
S(m):=\left\{a \in \mathcal{C}^{\infty}\left(\mathbb{R}^{2 n} ; \mathbb{R}\right)\left|\forall \alpha \in \mathbb{N}^{2 n}, \exists C_{\alpha}>0, \forall X \in \mathbb{R}^{2 n},\right| \partial^{\alpha} a(X) \mid \leqslant C_{\alpha} m(X)\right\} .
$$

One can find an order function $\tilde{m}$ such that $S(\tilde{m})=S(m)$ and $\tilde{m} \in S(\tilde{m})$; therefore we will always assume that the order function $m$ lies in its own class, i.e.

$$
m \in S(m) \text {. }
$$

Given an 'observable' $a \in S(m)$, its Weyl quantization is the operator

$$
\left[\mathrm{Op}_{\hbar}^{w}(a) \psi\right](x):=\frac{1}{(2 \pi \hbar)^{n}} \iint_{\mathbb{R}^{2 n}} e^{\frac{i}{\hbar}(x-y) \eta} a\left(\frac{x+y}{2}, \eta\right) \psi(y) \mathrm{d} y \mathrm{~d} \eta
$$

for $\psi \in \mathscr{S}\left(\mathbb{R}^{n}\right)$. Let $g:=\log (m)$ and $g^{w}:=\mathrm{Op}_{\hbar}^{w}(g)$; the exponential $e^{ \pm g^{w}}$ is a pseudodifferential operator with symbol $c_{ \pm} \in S(m)$.

Definition 2.3. The generalized Sobolev space associated with $m$ is given by

$$
\mathrm{H}_{\hbar}(m):=\left\{u \in \mathscr{S}^{\prime}\left(\mathbb{R}^{n}\right) \mid e^{g^{w}} u \in \mathrm{L}^{2}\left(\mathbb{R}^{n}\right)\right\}
$$

and the norm on $\mathrm{H}_{\hbar}(m)$ is defined by

$$
\|u\|_{\mathrm{H}_{\hbar}(m)}=\left\|e^{g^{w}} u\right\|_{\mathrm{L}^{2}\left(\mathbb{R}^{n}\right)} .
$$

We have the following equivalence of seminorms.

Lemma 2.4. Let $p$ be a Schwartz seminorm. One can find two order functions $m$ and $\tilde{m}$ satisfying $(2.2)$ and $C>0$ such that for any $u \in \mathscr{S}\left(\mathbb{R}^{n}\right)$ and $\hbar \in(0,1]$,

$$
\frac{1}{C}\|u\|_{\mathrm{H}_{\hbar}(m)} \leqslant p(u) \leqslant C\|u\|_{\mathrm{H}_{\hbar}(\tilde{m})}
$$

Operators with a symbol in $S(m)$ map the Schwartz space to itself and, more precisely, the continuity between semiclassical Sobolev spaces is given by the following proposition.

Proposition 2.5. Given two order functions $m_{1}, m_{2}$, if a $\in S\left(m_{1}\right)$, then

$$
\mathrm{Op}_{\hbar}^{w}(a): \mathrm{H}_{\hbar}\left(m_{2}\right) \rightarrow \mathrm{H}_{\hbar}\left(\frac{m_{2}}{m_{1}}\right)
$$

defines a continuous operator uniformly for $\hbar \in(0,1]$, such that as $\hbar \rightarrow 0$

$$
\left\|\mathrm{Op}_{\hbar}^{w}(a)\right\|_{\mathrm{H}_{\hbar}\left(m_{2}\right) \rightarrow \mathrm{H}_{\hbar}\left(m_{2} / m_{1}\right)}=\mathcal{O}(1) .
$$

The following well-known (and straightforward) lemma will be repeatedly used to deal with the $\sqrt{\hbar}$-localization of coherent states. 
Lemma 2.6. Let $c \in S(m)$, for some order function $m$ on $\mathbb{R}^{2 n}$. Then

$$
\left(T_{\hbar}(z) \Lambda_{\hbar}\right)^{-1} \mathrm{Op}_{\hbar}^{w}(c) T_{\hbar}(z) \Lambda_{\hbar}=\mathrm{Op}_{\hbar=1}^{w}\left(c_{1}\right),
$$

where $c_{1}(X):=c(z+\sqrt{\hbar} X)$ (and hence $\left.c_{1} \in S(m)\right)$.

We can now prove some propagator estimates.

Lemma 2.7. Let $z \in \mathbb{R}^{2 n}$ and $\hbar>0$. Then for any $f, \varphi \in \mathscr{S}\left(\mathbb{R}^{n}\right)$, for any order function $\nu$ on $\mathbb{R}^{2 n}$,

$$
\left\|T_{\hbar}(z) \Lambda_{\hbar} f\right\|_{\mathrm{H}_{\hbar}(\nu)} \leqslant C_{1}\|f\|_{\mathrm{H}_{1}(\nu)}
$$

and

$$
\left\|\left(T_{\hbar}(z) \Lambda_{\hbar}\right)^{-1} \varphi\right\|_{\mathrm{H}_{1}(\nu)} \leqslant C_{2}\|\varphi\|_{\mathrm{H}_{\hbar}(\nu)}
$$

with $C_{1}, C_{2}$ independent of $\hbar$, and $\mathrm{H}_{1}(\nu):=\mathrm{H}_{\hbar=1}(\nu)$.

Proof. Let $f, z, \nu$ as above. Let $\gamma:=\log (\nu)$, and $\gamma^{w}:=\mathrm{Op}_{\hbar}^{w}(\gamma), \gamma_{1}^{w}:=\mathrm{Op}_{\hbar=1}^{w}(\gamma)$. Then, using the unitarity of $T_{\hbar}(z) \Lambda_{\hbar}$,

$$
\left\|T_{\hbar}(z) \Lambda_{\hbar} f\right\|_{\mathrm{H}_{\hbar}(\nu)}=\left\|\left(T_{\hbar}(z) \Lambda_{\hbar}\right)^{-1} e^{\gamma^{w}} T_{\hbar}(z) \Lambda_{\hbar} e^{-\gamma_{1}^{w}} e^{\gamma_{1}^{w}} f\right\|_{L^{2}\left(\mathbb{R}^{n}\right)} .
$$

Since $e^{ \pm \gamma^{w}}=\mathrm{Op}_{\hbar}^{w}\left(c^{ \pm}\right)$with $c^{ \pm} \in S\left(\nu^{ \pm 1}\right)$, Lemma 2.6 gives

$$
\left(T_{\hbar}(z) \Lambda_{\hbar}\right)^{-1} e^{\gamma^{w}} T_{\hbar}(z) \Lambda_{\hbar}=\mathrm{Op}_{\hbar=1}^{w}\left(c_{1}\right)
$$

with $c_{1}: X \mapsto c(z+\sqrt{\hbar} X), c_{1} \in S(\nu)$. Therefore,

$$
\left(T_{\hbar}(z) \Lambda_{\hbar}\right) e^{\gamma^{w}} T_{\hbar}(z) \Lambda_{\hbar} e^{-\gamma_{1}^{w}}=\mathrm{Op}_{\hbar=1}^{w}\left(c_{1}\right) e^{-\gamma_{1}^{w}}
$$

is bounded on $L^{2}(\mathbb{R})$ because it is a pseudodifferential operator with a symbol in $S(1)$. Finally,

$$
\left\|T_{\hbar}(z) \Lambda_{\hbar} f\right\|_{\mathrm{H}_{\hbar}(\nu)} \leqslant\left\|\mathrm{Op}_{\hbar=1}^{w}\left(c_{1}\right) e^{-\gamma_{1}^{w}}\right\|_{\mathrm{L}^{2}\left(\mathbb{R}^{n}\right)}\|f\|_{\mathrm{H}_{1}(\nu)}
$$

For the reverse inequality, we can write

$$
\left\|\left(T_{\hbar}(z) \Lambda_{\hbar}\right)^{-1} \varphi\right\|_{\mathrm{H}_{1}(\nu)}=\left\|T_{\hbar}(z) \Lambda_{\hbar} e^{\gamma_{1}^{w}}\left(T_{\hbar}(z) \Lambda_{\hbar}\right)^{-1} e^{-\gamma^{w}} e^{\gamma^{w}} \varphi\right\|_{\mathrm{L}^{2}\left(\mathbb{R}^{n}\right)} .
$$

But,

with

$$
T_{\hbar}(z) \Lambda_{\hbar} e^{\gamma_{1}^{w}}\left(T_{\hbar}(z) \Lambda_{\hbar}\right)^{-1}=\mathrm{Op}_{\hbar}^{w}\left(c_{-1}\right)
$$

$$
c_{-1}: X \mapsto c\left(\frac{X-z}{\sqrt{\hbar}}\right)
$$

The symbol $c_{-1}$ belongs to the 'limit' class $S_{1 / 2}(\nu)$ (see [32, Chapitre 4] for a definition and the related theory). Thus, $\mathrm{Op}_{\hbar}^{w}\left(c_{-1}\right) \mathrm{Op}_{\hbar}^{w}\left(c^{-}\right)$is a pseudodifferential operator 
with a symbol in $S_{1 / 2}(1)$, and from the Calderon-Vaillancourt theorem it defines a bounded operator on $\mathrm{L}^{2}\left(\mathbb{R}^{n}\right)$. Finally,

$$
\left\|\left(T_{\hbar}(z) \Lambda_{\hbar}\right)^{-1} \varphi\right\|_{\mathrm{H}_{1}(\nu)} \leqslant\left\|\mathrm{Op}_{\hbar}^{w}\left(c_{-1}\right) e^{-\gamma^{w}}\right\|_{\mathrm{L}^{2}\left(\mathbb{R}^{n}\right)}\|\varphi\|_{\mathrm{H}_{\hbar}(\nu)},
$$

which concludes the proof.

We prove now the following continuity property of bounded propagators on generalized Sobolev spaces.

Lemma 2.8. Let $\left(H_{t}\right)_{t \in[0,1]}$ be a real-valued time-dependent family of Hamiltonians. We assume $\left(H_{t}\right)_{t \in[0,1]}$ to be continuous in time and to lie in $S(1)$ uniformly in times. Then there exists a family $\left(U_{\hbar}(t, s)\right)_{t, s \in[0,1]}$ of unitary operators on $\mathrm{L}^{2}\left(\mathbb{R}^{n}\right)$ satisfying

(1) $U_{\hbar}(0,0)=i d_{\mathrm{L}^{2}\left(\mathbb{R}^{n}\right)}$ and for any $r \in[0,1]$,

$$
U_{\hbar}(t, s)=U_{\hbar}(t, r) U_{\hbar}(r, s)
$$

(2) $U_{\hbar}(\cdot, \cdot)$ is strongly continuous on $\mathrm{L}^{2}\left(\mathbb{R}^{n}\right)$;

(3) for any $\psi \in \mathrm{L}^{2}\left(\mathbb{R}^{n}\right)$ and any $s \in[0,1]$, the map $t \mapsto U_{\hbar}(t, s) \psi$ is differentiable, and satisfies

$$
i \hbar \partial_{t} U_{\hbar}(t, s) \psi=\mathrm{Op}_{\hbar}^{w}\left(H_{t}\right) U_{\hbar}(t, s) \psi \quad \text { in } \mathrm{L}^{2}\left(\mathbb{R}^{n}\right),
$$

and this family is unique. Moreover, for any order function $\nu$, for any $\varphi \in \mathscr{S}\left(\mathbb{R}^{n}\right)$, we have

$$
\left\|U_{\hbar}(t, 0) \varphi\right\|_{\mathrm{H}_{\hbar}(\nu)} \leqslant C\|\varphi\|_{\mathrm{H}_{\hbar}(\nu)}
$$

where $C$ is $\hbar$-independent.

Proof. We consider the equation

$$
i \hbar \partial_{t} U_{\hbar}(t, 0) \varphi=\mathrm{Op}_{\hbar}^{w}\left(H_{t}\right) U_{\hbar}(t, 0) \varphi \quad \varphi \in \mathscr{S}\left(\mathbb{R}^{n}\right) .
$$

Let $\nu$ be an order function on $T^{*} \mathbb{R}^{n}$. Since $H_{t} \in S(1)$ uniformly for $t \in[0,1]$, $\mathrm{Op}_{\hbar}^{w}\left(H_{t}\right)$ defines a continuous linear operator on $\mathrm{H}_{\hbar}(\nu)$ with a uniform bound for $t \in[0,1]$. So, considering (2.4) as an EDO with values in $\mathrm{H}_{\hbar}(\nu)$, the CauchyLipschitz theorem gives us a unique solution $\left(U_{\hbar}(t, 0) \varphi\right) \in \mathcal{C}^{1}\left([0,1], \mathrm{H}_{\hbar}(\nu)\right)$, with $\left(U_{\hbar}(t, s)\right)_{(t, s) \in[0,1]}$ satisfying the above assumptions (1), (2) and (3). One should remark that $U_{\hbar}(t, 0)$ then defines an operator in $\mathcal{C}^{1}\left([0,1], \mathcal{L}\left(\mathrm{H}_{\hbar}(\nu)\right)\right)$, that is unitary for $\nu=1$, i.e. on $\mathrm{L}^{2}\left(\mathbb{R}^{n}\right)$. In order to prove the announced estimate, let now $\gamma:=\log (\nu)$ and $\gamma^{w}:=\mathrm{Op}_{\hbar}^{w}(\gamma)$. We set also $U_{\hbar}^{\gamma}:=e^{\gamma^{w}} U_{\hbar} e^{-\gamma^{w}}$, and $\hat{H}_{t}^{\gamma}:=e^{\gamma^{w}} \mathrm{Op}_{\hbar}^{w}\left(H_{t}\right) e^{-\gamma^{w}}$, so that $U_{\hbar}^{\gamma} \in \mathcal{C}^{1}\left([0,1], \mathcal{L}\left(\mathrm{L}^{2}\left(\mathbb{R}^{n}\right)\right)\right)$ and $\hat{H}_{t}^{\gamma}$ is a pseudodifferential operator with symbol in $S(1)$ such that

$$
i \hbar \partial_{t} U_{\hbar}^{\gamma}(t, 0)=\hat{H}_{t}^{\gamma} U_{\hbar}^{\gamma}(t, 0) \quad \text { and } \quad-i \hbar \partial_{t} U_{\hbar}^{\gamma}(t, 0)^{*}=U_{\hbar}^{\gamma}(t, 0)^{*} \hat{H}_{t}^{-\gamma} \text {. }
$$


Then we have for $\varphi \in \mathrm{L}^{2}\left(\mathbb{R}^{n}\right)$,

$$
\begin{aligned}
i \hbar \partial_{t}\left\|U_{\hbar}^{\gamma}(t, 0) \varphi\right\|_{\mathrm{L}^{2}\left(\mathbb{R}^{n}\right)}^{2} & =\left\langle U_{\hbar}^{\gamma}(t, 0)^{*} e^{-\gamma^{w}}\left[e^{2 \gamma^{w}}, \mathrm{Op}_{\hbar}^{w}\left(H_{t}\right)\right] e^{-\gamma^{w}} U_{\hbar}^{\gamma}(t, 0) \varphi_{0}, \varphi_{0}\right\rangle_{\mathrm{L}^{2}\left(\mathbb{R}^{n}\right)} \\
& =\hbar\left\langle\eta^{w}(t) U_{\hbar}^{\gamma}(t, 0) \varphi, U_{\hbar}^{\gamma}(t, 0) \varphi\right\rangle_{\mathrm{L}^{2}\left(\mathbb{R}^{n}\right)}
\end{aligned}
$$

where $\eta^{w}(t):=\mathrm{Op}_{\hbar}^{w}(\eta(t))=\frac{i}{\hbar} e^{-\gamma^{w}}\left[e^{2 \gamma^{w}}, \mathrm{Op}_{\hbar}^{w}\left(H_{t}\right)\right] e^{-\gamma^{w}}$ whose symbol $\eta(t)$ belongs to $S(1)$ uniformly in time. Therefore $\eta^{w}(t) U_{\hbar}^{\gamma}(t, 0) \varphi \in \mathrm{L}^{2}\left(\mathbb{R}^{n}\right)$, which justifies the above computation. Then by Proposition 2.5, we have

$$
\partial_{t}\left\|U_{\hbar}^{\gamma}(t, 0) \varphi\right\|_{\mathrm{L}^{2}\left(\mathbb{R}^{n}\right)}^{2} \leqslant\left\|\eta^{w}(t)\right\|_{\mathrm{L}^{2}\left(\mathbb{R}^{n}\right) \rightarrow \mathrm{L}^{2}\left(\mathbb{R}^{n}\right)}\left\|U_{\hbar}^{\gamma}(t, 0) \varphi\right\|_{\mathrm{L}^{2}\left(\mathbb{R}^{n}\right)}^{2}
$$

which implies

$$
\left\|U_{\hbar}^{\gamma}(t, 0) \varphi\right\|_{\mathrm{L}^{2}\left(\mathbb{R}^{n}\right)} \leqslant\left(\exp \int_{0}^{1}\left\|\eta^{w}(s)\right\|_{\mathrm{L}^{2}\left(\mathbb{R}^{n}\right) \rightarrow \mathrm{L}^{2}\left(\mathbb{R}^{n}\right)} \mathrm{d} s\right)\|\varphi\|_{\mathrm{L}^{2}\left(\mathbb{R}^{n}\right)} .
$$

Considering now $\psi \in \mathrm{H}_{\hbar}(\nu)$ and $\varphi=e^{\gamma^{w}} \psi$ proves the result.

We now turn to the propagation of coherent states for finite times. The following proposition generalizes to the class $\mathfrak{C}^{[n]}$ the result obtained by Robert for Gaussian states (see [6, Section 4.3.1]).

Proposition 2.9. Let $\left(H_{t}\right)_{t \in[0,1]}$ and the associated propagator $\left(U_{\hbar}(t, s)\right)_{t, s \in[0,1]}$ be as in Lemma 2.8. Consider the associated Hamiltonian flow $\Phi_{H}:[0,1] \times \mathbb{R}^{2 n} \rightarrow \mathbb{R}^{2 n}$. Then, for a coherent state $\varphi_{\hbar} \in \mathfrak{C}^{[n]}$ centered at $z_{0} \in \mathbb{R}^{2 n}$, the state $\psi_{\hbar}:=U_{\hbar}(1,0) \varphi_{\hbar}$ is still a coherent state in $\mathfrak{C}^{[n]}$, centered at $z_{1}:=\Phi_{H}^{t=1}\left(z_{0}\right)$.

Proof. Let

$$
\varphi_{\hbar}(t)=U_{\hbar}(t, 0) \varphi_{\hbar} \quad \text { and } \quad \psi_{\hbar}=\varphi_{\hbar}(1) .
$$

Since $\varphi_{\hbar} \in \mathscr{S}\left(\mathbb{R}^{n}\right)$, from Lemma 2.8 we know that, for all $t$ in $[0,1], \varphi_{\hbar}(t) \in \mathscr{S}\left(\mathbb{R}^{n}\right)$. Because $\varphi_{\hbar} \in \mathfrak{C}^{[n]}$, there are $\mathrm{f}_{\hbar}^{0} \in \mathfrak{s}^{[n]}, \delta_{0} \in \mathbb{R}$ and $z_{0} \in \mathbb{R}^{2 n}$ such that

$$
\varphi_{\hbar}=e^{-i \delta_{0} / \hbar} T_{\hbar}\left(z_{0}\right) \Lambda_{\hbar}\left(f+\hbar^{\beta} g_{\hbar}\right),
$$

$\beta>0, f \in \mathscr{S}\left(\mathbb{R}^{n}\right)$ and $\left(g_{\hbar}\right)_{\hbar \in\left(0, \hbar_{0}\right)} \subset \mathscr{S}\left(\mathbb{R}^{n}\right)$ (see Definition 2.2). Let $\left(z_{t}\right)_{0 \leqslant t \leqslant 1}$ denote the classical evolution from $z_{0}$ led by the time-dependent Hamiltonian $\left(H_{t}\right)_{0 \leqslant t \leqslant 1}$,

$$
\forall t \in[0,1], \quad z_{t}=\Phi_{H}^{t}\left(z_{0}\right) .
$$

As $\left(H_{t}\right)_{t \in[0,1]}$ lies uniformly in times in $S(1)$, the corresponding Hamiltonian vector field is uniformly bounded in times, that ensures us the global existence of the flow $\left(\Phi_{H}^{t}\right)_{t \in[0,1]}$. Introducing the action integral

$$
\delta_{t}:=\delta_{0}+\int_{0}^{t}\left(p_{s} \cdot \dot{q}_{s}-H_{s}\left(z_{s}\right)\right) \mathrm{d} s-\frac{1}{2}\left(q_{t} \cdot p_{t}-q_{0} \cdot p_{0}\right),
$$


we define

$$
v_{\hbar}(t):=e^{-i \delta_{t} / \hbar} \Lambda_{\hbar}^{-1} T_{\hbar}\left(-z_{t}\right) U_{\hbar}(t, 0) \varphi_{\hbar}(0) .
$$

We establish the propagation equation of $v_{\hbar}(t)$. Noting that $\forall \psi \in \mathscr{S}\left(\mathbb{R}^{n}\right)$,

$$
\partial_{t}\left(T\left(z_{t}\right) \psi\right)(x)=\left[T_{\hbar}\left(-z_{t}\right)\left(-\frac{i}{\hbar}\left(\frac{\dot{q}_{t} p_{t}-q_{t} \dot{p}_{t}}{2}+\dot{p}_{t} \cdot x+\dot{q}_{t} \cdot i \hbar \nabla_{x}\right)\right) \psi\right](x)
$$

and by the differentiability property (2.3),

$$
\begin{aligned}
i \partial_{t} v_{\hbar}(t)=\frac{1}{\hbar}\left[\Lambda_{\hbar}^{-1} T_{\hbar}\left(-z_{t}\right) \mathrm{Op}_{\hbar}^{w}\left(H_{t}\right) T_{\hbar}\left(z_{t}\right) \Lambda_{\hbar}\right. & \\
& \left.\quad-\Lambda_{\hbar}^{-1}\left(\mathrm{Op}_{\hbar}^{w}(x) \partial_{q} H_{t}\left(z_{t}\right)-\mathrm{Op}_{\hbar}^{w}(\xi) \partial_{p} H_{t}\left(z_{t}\right)\right) \Lambda_{\hbar}-H_{t}\left(z_{t}\right)\right] v_{\hbar}(t)
\end{aligned}
$$

where $(x, \xi)=X \in \mathbb{R}^{2 n}$. Since

$$
\Lambda_{\hbar}^{-1} T_{\hbar}\left(-z_{t}\right) \mathrm{Op}_{\hbar}^{w}\left(H_{t}\right) T_{\hbar}\left(z_{t}\right) \Lambda_{\hbar}=\mathrm{Op}_{\hbar}^{w}\left(X \mapsto H_{t}\left(z_{t}+\sqrt{\hbar} X\right)\right),
$$

we are naturally led to Taylor expanding the function $X \mapsto H_{t}\left(z_{t}+\sqrt{\hbar} X\right)$ around the point $z_{t}$; let

$$
K_{2}(t, X):=X^{\mathbf{T}} \operatorname{Hess}\left(H_{t}, z_{t}\right) X
$$

and let $R_{\hbar}^{(3)}$ be the integral remaining term of order 3 in this Taylor expansion. We obtain

$$
\left\{\begin{array}{l}
i \partial_{t} v_{\hbar}(t)=\mathrm{Op}_{\hbar=1}^{w}\left(K_{2}(t, X)+\sqrt{\hbar} R_{\hbar}^{(3)}(t, X)\right) v_{\hbar}(t) \\
v_{\hbar}(t=0)=f+\hbar^{\beta} g_{\hbar}
\end{array}\right.
$$

Recall, from the definition $(2.6)$ of $v_{\hbar}(t)$, that this equation admits a propagator $P_{1}(\cdot, \cdot)$ given by

$$
P_{1}:(t, s) \in[0,1]^{2} \mapsto P_{1}(t, s)=\left(T_{\hbar}\left(z_{t}\right) \Lambda_{\hbar}\right)^{-1} U_{\hbar}(t, s) T_{\hbar}\left(z_{t}\right) \Lambda_{\hbar} .
$$

From this formula, $P_{1}$ inherits the group and strong continuity properties of $U_{\hbar}$. Moreover, it defines a map from $\mathscr{S}\left(\mathbb{R}^{n}\right)$ to itself that satisfies, for any $v \in \mathscr{S}\left(\mathbb{R}^{n}\right)$,

$$
i \partial_{t} P_{1}(t, 0) v=\mathrm{Op}_{\hbar=1}^{w}\left(K_{2}+\sqrt{\hbar} R_{\hbar}^{(3)}\right) v
$$

and from Lemma 2.7 and Lemma 2.8, for any order function $\nu$,

$$
\left\|P_{1}(t, 0) v\right\|_{\mathrm{H}_{1}(\nu)} \leqslant C\|v\|_{\mathrm{H}_{1}(\nu)},
$$

where $C$ is time and $\hbar$ independent. We consider now the following propagation equation in $v^{(0)}$ :

$$
\left\{\begin{array}{l}
i \partial_{t} v^{(0)}(t)=\mathrm{Op} \\
v^{(0)}(t=0)=f
\end{array}\right.
$$


Since it is defined by a time-dependent quadratic Hamiltonian, we may apply the result of [5, Theorem 2.8], which asserts that the propagator is well-defined as long as the classical flow $z_{t}$ exists. Hence for $t \in[0,1]$, Equation (2.7) admits a propagator $\left(P_{q}(t, s)\right)_{t, s \in[0,1]}$ (where the subscript $q$ stands for 'quadratic'), which is smooth in $t$ and unitary on $\mathrm{L}^{2}\left(\mathbb{R}^{n}\right)$, satisfying

$$
P_{q}(t, r) P_{q}(r, s)=P_{q}(t, s) .
$$

In order to prove that $P_{q}$ acts on $\mathscr{S}\left(\mathbb{R}^{n}\right)$, we will use Gaussian coherent states. Let $G_{0}$ be the normalized Gaussian on $\mathbb{R}^{n}$,

$$
G_{0}(X)=\frac{1}{\pi^{n / 4}} e^{-\frac{X \cdot X}{2}}
$$

and define

$$
G_{\tilde{z}}:=T_{\hbar=1}(\tilde{z}) G_{0} .
$$

Since $P_{q}(t, 0) f \in \mathrm{L}^{2}\left(\mathbb{R}^{n}\right)$, the resolution of identity property of Gaussian coherent states gives

$$
P_{q}(t, 0) f=\int_{\mathbb{R}^{2 n}}\left\langle f, P_{q}(t, 0)^{*} G_{\tilde{z}}\right\rangle G_{\tilde{z}} \mathrm{~d} \tilde{z} .
$$

From [6, Chapter 3, Theorem 16], there is a differentiable map

$$
t \in[0,1] \mapsto \Gamma_{t} \in \Pi_{n}^{+}
$$

where $\Pi_{n}^{+}$is the Siegel space of complex and symmetric matrices with positive-definite imaginary part, such that,

$$
P_{q}(t, 0) G_{\tilde{z}}=G_{\tilde{z} t}^{\Gamma_{t}}
$$

Here we have denoted, for $\Gamma \in \Pi_{n}^{+}$,

$$
G_{0}^{\Gamma}(X)=a_{\Gamma} e^{-i \frac{X \cdot \Gamma X}{2}},
$$

where $a_{\Gamma}$ is the $\mathrm{L}^{2}$-normalization constant and $\tilde{z}_{t}$ corresponds to the Hamiltonian flow of the quadratic Hamiltonian starting from $\tilde{z}$. Since $f \in \mathscr{S}\left(\mathbb{R}^{n}\right)$ we can, for any $t \in[0,1]$, integrate by parts and obtain, for any integer $N>0$

$$
\left\langle f, G_{\tilde{z}_{t}}\right\rangle_{\mathrm{L}^{2}} \leqslant \frac{C_{N}\left(f, \Gamma_{t}\right)}{\left\langle\tilde{z}_{t}\right\rangle^{N}} .
$$

This ensures, from usual theorems of derivation under the integral (2.8), that

$$
[0,1] \ni t \mapsto P_{q}(t, 0) f \in \mathscr{S}\left(\mathbb{R}^{n}\right)
$$

is continuous. We now prove that such a quadratic propagator satisfies the Schwartz estimates as in Lemma 2.8. To do so, considering $\nu$ an order function, $\gamma:=\log (\nu)$, 
and $\gamma_{1}^{w}:=\mathrm{Op}_{\hbar=1}^{w}(\gamma)$, we have for any Schwartz function $f$,

$$
\left\|P_{q}(t, 0) f\right\|_{\mathrm{H}_{1}(\nu)}=\left\|e^{\gamma_{1}^{w}} P_{q}(t, 0) f\right\|_{\mathrm{L}^{2}\left(\mathbb{R}^{n}\right)} \leqslant \iint_{\mathbb{R}^{2 n}}\left|\left\langle f, G_{\tilde{z}_{t}}^{\Gamma_{t}}\right\rangle\right|\left\|e^{\gamma_{1}^{w}} G_{\tilde{z}}\right\|_{\mathrm{L}^{2}\left(\mathbb{R}^{n}\right)} \mathrm{d} \tilde{z} .
$$

A computation gives (see Proposition 2.12) that there is an integer $l$ depending only on the order function $\nu$ such that

$$
\left\|e^{\gamma_{1}^{w}} G_{\tilde{z}}\right\|_{L^{2}\left(\mathbb{R}^{n}\right)} \leqslant C\langle\tilde{z}\rangle^{l}
$$

where $C$ is a constant depending only on $\nu$; hence one can find an order function $\tilde{\nu}$ such that

$$
\left\|P_{q}(t, 0) f\right\|_{\mathrm{H}_{1}(\nu)} \leqslant C(\nu)\|f\|_{\mathrm{H}_{1}(\tilde{\nu})} .
$$

So, Equation (2.7) admits a propagator family $\left(P_{q}(t, s)\right)_{t, s \in[0,1]}$ that satisfies the same Schwartz continuity property as $P_{1}$. For $t \in[0,1]$, let

$$
v^{(0)}(t):=P_{q}(t, 0) f \in \mathscr{S}\left(\mathbb{R}^{n}\right)
$$

be the solution to (2.7); notice that $v^{(0)}(t)$ does not depend on $\hbar$ and satisfies, for any $t \in[0,1],\left\|v^{(0)}(t)\right\|_{\mathrm{L}^{2}\left(\mathbb{R}^{n}\right)}=\left\|v^{(0)}(0)\right\|_{\mathrm{L}^{2}\left(\mathbb{R}^{n}\right)}$. Using now Duhamel's principle, we get

$$
v_{\hbar}(t)-v^{(0)}(t)=\hbar^{\beta} P_{1}(t, 0) g_{\hbar}-i \sqrt{\hbar} \int_{0}^{t} P_{1}(t, s) \mathrm{Op}_{\hbar=1}^{w}\left(R_{\hbar}^{(3)}\right) P_{q}(s, 0) f \mathrm{~d} s .
$$

From Lemma 2.8, as the Schwartz seminorms of $\left(g_{\hbar}\right)_{\hbar>0}$ are bounded, the same holds for $\left(P_{1}(t, 0) g_{\hbar}\right)_{\hbar>0}$ for any $t \in[0,1]$. Since $H_{t} \in S(1)$, it follows from Taylor's formula that $R_{\hbar}^{(3)} \in S\left(\langle X\rangle^{2}\right)$, uniformly for $t \in[0,1]$. Therefore we may apply Lemma 2.4 and Proposition 2.5 to conclude, from the continuity of $\left(P_{1}(t, s)\right)_{t, s \in[0,1]}$ and $\left(P_{q}(s, 0)\right)_{s \in[0,1]}$ with respect to $(t, s)$ in $[0,1]$, that the integral

$$
\int_{0}^{t} P_{1}(t, s) \mathrm{Op}_{\hbar=1}^{w}\left(R_{\hbar}^{(3)}\right) P_{q}(s, 0) f \mathrm{~d} s
$$

is uniformly bounded in $\mathscr{S}\left(\mathbb{R}^{n}\right)$ for all $t \in[0,1]$ and $\hbar \in(0,1]$. Finally, we have

$$
v_{\hbar}(1)=v^{(0)}(1)+\hbar^{\tilde{\beta}} \tilde{g}_{\hbar}
$$

where

$$
\tilde{g}_{\hbar}:=\hbar^{\beta-\tilde{\beta}} P_{1}(t, 0) g_{\hbar}-i \hbar^{1 / 2-\tilde{\beta}} \int_{0}^{t} P_{1}(t, s) \mathrm{Op}_{\hbar=1}^{w}\left(R_{\hbar}^{(3)}\right) P_{q}(s, 0) f \mathrm{~d} s
$$

and $\tilde{\beta}:=\min (1 / 2, \beta)$, which proves $v_{\hbar}(1) \in \mathfrak{s}^{[n]}$ (actually we obtain $v_{\hbar}(t) \in \mathfrak{s}^{[n]}$ for any $t \in[0,1])$. Finally,

$$
\psi_{\hbar}=e^{i \delta_{1} / \hbar} T_{\hbar}\left(z_{1}\right) \Lambda_{\hbar} v_{\hbar}(1)
$$


where $\delta_{1}:=\delta_{t=1}$ and $z_{1}:=z_{t=1}$, which establishes the announced result.

Remark 2.10. The fact that the function $f$ in (2.5) is normalized in $\mathrm{L}^{2}$ is not relevant. Indeed, the proof still works for any shape function of the form $\mathrm{f}_{\hbar}=f+\hbar^{\beta} g_{\hbar}$, where $f$ is an $\hbar$-independent function of $\mathrm{L}^{2}\left(\mathbb{R}^{2}\right), \beta>0$ and $\left(g_{\hbar}\right)_{\hbar>0}$ is a family of functions in $\mathscr{S}\left(\mathbb{R}^{2}\right)$ with bounded seminorms, uniformly with respect to $\hbar \in(0,1]$.

We now develop some very useful tools on the study of the coherent state class we introduced earlier on, that is the Wigner transform.

2.3. Wigner transform of coherent states and applications. The main idea of the Wigner transform is to take advantage of the strong localization of coherent states (sometimes called 'peak states') to get estimates on pseudodifferential operators from the associated symbol. We refer to [9] for the definition below of the Wigner transform. In [28, 6], authors use fast decay properties of the Wigner transform of coherent states to get strong approximation results on the propagation of coherent states depending on the regularity of the generator of the propagation. Here, we will take advantage of the Schwartz property of our class of coherent states to prove some estimates on pseudodifferential operators for symbols class $S(m)$ for arbitrary large order functions $m$.

We consider a family $\left(\varphi_{z}\right)_{z \in \mathbb{R}^{2 n}}$ of coherent states with the same shape: for any $z \in \mathbb{R}^{2 n}, \varphi_{z}=T_{\hbar}(z) \Lambda_{\hbar} \mathrm{f}_{\hbar}$, where $\mathrm{f}_{\hbar}$ is a given shape in $\mathfrak{s}^{[n]}$. Now, let $a \in S(m)$ a given symbol, with $m(z) \leqslant\langle z\rangle^{l}$ for an integer $l$. We have

$$
\begin{aligned}
& \left\langle\mathrm{Op}_{\hbar}^{w}(a) \varphi_{z}, \varphi_{z^{\prime}}\right\rangle_{\mathrm{L}^{2}\left(\mathbb{R}^{n}\right)}=\frac{2^{n}}{(2 \pi \hbar)^{n}} \times \\
& \quad \int_{\mathbb{R}^{n}} \iint_{\mathbb{R}^{2 n}} e^{\frac{2 i}{\hbar}(x-\tilde{y}) \eta} a(\tilde{y}, \eta) e^{-\frac{i}{2 \hbar} q p+\frac{i}{\hbar} p(2 \tilde{y}-x)} \mathrm{f}_{\hbar}\left(\frac{2 \tilde{y}-x-q}{\sqrt{\hbar}}\right) \mathrm{d} \tilde{y} \mathrm{~d} \eta e^{\frac{i}{2 \hbar} q^{\prime} p^{\prime}-\frac{i}{\hbar} p^{\prime} x} \overline{\mathrm{f}_{\hbar}\left(\frac{x-q^{\prime}}{\sqrt{\hbar}}\right)} \mathrm{d} x,
\end{aligned}
$$

which leads to the following definition.

Definition 2.11. We define the Wigner function $\mathcal{W}_{z, z^{\prime}}$ associated with the family $\left(\varphi_{z}\right)_{z \in \mathbb{R}^{2 n}}$ of coherent states with shape $\mathrm{f}_{\hbar} \in \mathfrak{s}^{[n]}$ as

$$
\begin{aligned}
\mathcal{W}_{z, z^{\prime}}(y, \eta)= & \frac{2^{n}}{\hbar^{n / 2}} e^{\frac{i}{\hbar} \omega\left(Y-\frac{1}{2} z^{\prime}, z-z^{\prime}\right)} \times \\
& \int_{\mathbb{R}^{n}} e^{\frac{2 i}{\hbar} u\left(\eta-\frac{p+p^{\prime}}{2}\right)} \mathrm{f}_{\hbar}\left(\frac{y-\frac{q+q^{\prime}}{2}-u}{\sqrt{\hbar}}\right) \overline{\mathrm{f}_{\hbar}\left(\frac{y-\frac{q+q^{\prime}}{2}+u}{\sqrt{\hbar}}\right)} \mathrm{d} u
\end{aligned}
$$

where $\omega$ is the canonical symplectic form on $\mathbb{R}^{2 n}$

$$
\omega((a, b),(c, d))=b \cdot c-a \cdot d
$$


and where $Y:=(y, \eta)$.

With this definition we have the following formula

$$
\left\langle\mathrm{Op}_{\hbar}^{w}(a) \varphi_{z}, \varphi_{z^{\prime}}\right\rangle_{\mathrm{L}^{2}\left(\mathbb{R}^{n}\right)}=\frac{1}{(2 \pi \hbar)^{n}} \int_{\mathbb{R}^{2 n}} a(Y) \mathcal{W}_{z, z^{\prime}}(Y) \mathrm{d} Y
$$

which highlights the Wigner function as a Kernel to compute the action of pseudodifferential operators on coherent states. Setting

$$
\Psi_{\hbar}:=(\hat{y}, u) \in \mathbb{R}^{2 n} \mapsto \mathrm{f}_{\hbar}(\hat{y}-u) \overline{\mathrm{f}_{\hbar}(\hat{y}+u)},
$$

$\Psi_{\hbar}$ is a Schwartz function on $\mathbb{R}^{2 n}$ with all Schwartz seminorm uniformly bounded with $\hbar \in\left(0, \hbar_{0}\right)$ and we have the shorter expression

$$
\mathcal{W}_{z, z^{\prime}}(y, \eta)=\frac{2^{n}}{\hbar^{n / 2}} e^{\frac{i}{\hbar} \omega\left(Y-\frac{1}{2} z^{\prime}, z-z^{\prime}\right)} \times \int_{\mathbb{R}^{n}} e^{\frac{2 i}{\hbar} u\left(\eta-\frac{p+p^{\prime}}{2}\right)} \Psi_{\hbar}\left(\frac{u}{\sqrt{\hbar}}, \frac{y-\frac{q+q^{\prime}}{2}}{\sqrt{\hbar}}\right) \mathrm{d} u
$$

The goal is to use the oscillating integral structure to show some fast decay with respect to $Y-\frac{z+z^{\prime}}{2}$ and $z-z^{\prime}$ and to prove the following key property for this article.

Proposition 2.12. Let $a \in S(m)$ for an order function $m$ on $\mathbb{R}^{2 n}$ satisfying for $l \in \mathbb{N}, C>0$,

$$
\forall Y \in \mathbb{R}^{2 n},|m(Y)| \leqslant C\langle Y\rangle^{l} .
$$

Let $\left(\varphi_{z}\right)_{z \in \mathbb{R}^{2 n}}$ being a family of coherent states with shape $\mathrm{f}_{\hbar} \in \mathfrak{s}^{[n]}$. Then we have for $\varepsilon>0$, for $N, M \in \mathbb{N}$ large enough,

$$
\begin{aligned}
& \left\|\mathrm{Op}_{\hbar}^{w}(a) \varphi_{z}\right\|_{\mathrm{L}^{2}\left(\mathbb{R}^{n}\right)}^{2} \leqslant \hbar^{-3 n / 2} C_{\varepsilon}\left(\mathrm{f}_{\hbar}\right) \\
& \qquad \mathrm{f}_{\hbar} \|_{\mathrm{L}^{2}\left(\mathbb{R}^{n}\right)}^{2-\varepsilon} \times \\
& \qquad \int_{\mathbb{R}^{2 n}} \iint_{\operatorname{supp}(a)}\left\langle\frac{Y-\frac{z+z^{\prime}}{2}}{\sqrt{\hbar}}\right\rangle^{-N} \mathrm{~d} Y\left\langle\frac{z-z^{\prime}}{\sqrt{\hbar}}\right\rangle^{-M} \mathrm{~d} z^{\prime}
\end{aligned}
$$

with

$$
C_{\varepsilon}\left(\mathrm{f}_{\hbar}\right)=\max _{\alpha \in \mathbb{N}^{n},|\alpha| \leqslant K}\left(\left\|\partial_{\alpha} a(Y)\langle Y\rangle^{-l}\right\|_{\mathrm{L}^{\infty}\left(\mathbb{R}^{n}\right)}\right) \sum_{i \in I} \prod_{j \in J} p_{i, j}\left(\mathrm{f}_{\hbar}\right)^{\delta_{i, j}}
$$

where $K$ is an integer depending on $N$ and $M$ and $I$, $J$ finite sets depending only on $N, M, l$ and $\varepsilon$, and with $p_{i, j}$ being some Schwartz seminorm, $\delta_{i, j}>0$ depending on $\varepsilon$.

Remark 2.13. The parameter $\varepsilon$ is necessary to handle the growth of the symbol $a$ at infinity, which is compensated by $\mathrm{f}_{\hbar}^{\varepsilon}$. Of course, if a $\in S(1)$ we don't need this analysis, and the result holds for $\varepsilon=0$, directly from the Calderon-Vaillancourt theorem. 
Proof. Applying Plancherel's theorem along with (2.9), we first get

$$
\left\|\mathrm{Op}_{\hbar}^{w}(a) \varphi_{z}\right\|_{\mathrm{L}^{2}\left(\mathbb{R}^{n}\right)}^{2}\left\|\mathrm{f}_{\hbar}\right\|_{\mathrm{L}^{2}\left(\mathbb{R}^{n}\right)}^{2}=\frac{1}{(2 \pi \hbar)^{3 n}} \iint_{\mathbb{R}^{2 n}}\left|\iint_{\mathbb{R}^{2 n}} a(Y) \mathcal{W}_{z, z^{\prime}}(Y) \mathrm{d} Y\right|^{2} \mathrm{~d} z^{\prime} .
$$

We now use the fact that the Wigner function is an oscillating integral. Noting that

$$
\left\langle\frac{z-z^{\prime}}{\sqrt{\hbar}}\right\rangle^{-2 N} \cdot\left(1-\hbar \Delta_{Y}\right)^{N} \cdot e^{\frac{i}{\hbar} \omega\left(Y-\frac{z^{\prime}}{2}, z-z^{\prime}\right)}=e^{\frac{i}{\hbar} \omega\left(Y-\frac{z^{\prime}}{2}, z-z^{\prime}\right)}
$$

and, for any integer $M$,

$$
\left\langle\frac{\eta-\frac{p+p^{\prime}}{2}}{\sqrt{\hbar}}\right\rangle^{-2 M} \cdot\left(1-\hbar \Delta_{u}\right)^{M} e^{\frac{2 i}{\hbar} u \cdot\left(\eta-\frac{p+p^{\prime}}{2}\right)}=2^{M} e^{\frac{2 i}{\hbar} u \cdot\left(\eta-\frac{p+p^{\prime}}{2}\right)}
$$

we get

$$
\begin{aligned}
\left\|\mathrm{Op}_{\hbar}^{w}(a) \varphi_{z}\right\|_{\mathrm{L}^{2}\left(\mathbb{R}^{n}\right)}^{2}\left\|\mathrm{f}_{\hbar}\right\|_{\mathrm{L}^{2}\left(\mathbb{R}^{n}\right)}^{2} \leqslant & \frac{1}{(2 \pi \hbar)^{3 n}} \cdot \frac{2^{2 n}}{\hbar^{n}} \times \\
\iint_{\mathbb{R}^{2 n}} & {\left[\iint_{\mathbb{R}^{2 n}} \frac{\langle Y\rangle^{l}}{\left\langle\frac{z-z^{\prime}}{\sqrt{\hbar}}\right\rangle^{2 N}\left\langle\frac{Y-\frac{z+z^{\prime}}{2}}{\sqrt{\hbar}}\right\rangle^{2 M}} \sum_{|k|=0}^{2 N} 2^{2 M} C_{k}\langle Y\rangle^{-l}\left|\partial_{Y}^{k} a(Y)\right| \times\right.} \\
& \left.\int_{\mathbb{R}^{n}}\left\langle\frac{y-\frac{q+q^{\prime}}{2}}{\sqrt{\hbar}}\right\rangle^{2 M}\left|\left(1-\hbar \Delta_{u}\right)^{M} \Psi_{\hbar}^{[k]}\left(\frac{u}{\sqrt{\hbar}}, \frac{y-\frac{q+q^{\prime}}{2}}{\sqrt{\hbar}}\right)\right| \mathrm{d} u \mathrm{~d} Y\right]^{2} \mathrm{~d} z^{\prime}
\end{aligned}
$$

with

$$
\Psi_{\hbar}^{[k]}\left(\frac{u}{\sqrt{\hbar}}, \frac{y-\frac{q+q^{\prime}}{2}}{\sqrt{\hbar}}\right)=\sum_{0 \leqslant\left|j_{k}^{1}\right|,\left|j_{k}^{2}\right| \leqslant 2 N} \tilde{C}_{k}\left(\frac{u}{\sqrt{\hbar}}\right)^{j_{k}^{1}} \partial_{y}^{j_{k}^{2}} \Psi_{\hbar}\left(\frac{u}{\sqrt{\hbar}}, \frac{y-\frac{q+q^{\prime}}{2}}{\sqrt{\hbar}}\right)
$$

constants $\tilde{C}_{k}$ not depending on $\hbar$, for $k \in \mathbb{N}^{2 n}$. As $a \in S(m)$, with $\forall Y \in \mathbb{R}^{2 n}$ $|m(Y)| \leqslant\langle Y\rangle^{l}$, we have

$$
\sup _{0 \leqslant|k| \leqslant N}\left\|2^{2 M} C_{k} \partial_{Y}^{k} a(Y)\langle Y\rangle^{-l}\right\|_{L^{\infty}(\mathrm{d} Y)} \leqslant C(l, M)
$$

with $C(l, M)$ independent of $\hbar$. Moreover,

$$
\frac{\langle Y\rangle^{l}}{\left\langle\frac{z-z^{\prime}}{\sqrt{\hbar}}\right\rangle^{2 N}\left\langle\frac{Y-\frac{z+z^{\prime}}{2}}{\sqrt{\hbar}}\right\rangle^{2 M}} \leqslant \frac{C^{l}\langle z\rangle^{l}}{\left\langle\frac{z-z^{\prime}}{2}\right\rangle^{2 N-l}\left\langle\frac{Y-\frac{z+z^{\prime}}{\sqrt{\hbar}}}{\sqrt{\hbar}}\right\rangle^{2 M-l}}
$$


Finally, setting

$$
\begin{aligned}
& C_{l, M}(Y)=C(k, l, M) \times \\
& \qquad \sum_{|k|=0}^{2 N} \int_{\mathbb{R}^{n}}\left\langle\frac{y-\frac{q+q^{\prime}}{2}}{\sqrt{\hbar}}\right\rangle^{2 M}\left|\left(1-\hbar \Delta_{u}\right)^{M} \Psi_{\hbar}^{[k]}\left(\frac{u}{\sqrt{\hbar}}, \frac{y-\frac{q+q^{\prime}}{2}}{\sqrt{\hbar}}\right)\right| \mathrm{d} u,
\end{aligned}
$$

from Hölder inequality in $Y$ variable, we get

$$
\begin{aligned}
& \left\|\mathrm{Op}_{\hbar}^{w}(a) \varphi_{z}\right\|_{\mathrm{L}^{2}\left(\mathbb{R}^{n}\right)}^{2}\left\|\mathrm{f}_{\hbar}\right\|_{\mathrm{L}^{2}\left(\mathbb{R}^{n}\right)}^{2} \leqslant C^{2 l}\langle z\rangle^{2 l} \cdot \frac{2^{2 n}}{(2 \pi \hbar)^{3 n} \hbar^{n}} \times \\
& \iint_{\mathbb{R}^{2 n}}\left[\iint_{\operatorname{supp}(a)}\left(\left\langle\frac{z-z^{\prime}}{\sqrt{\hbar}}\right\rangle^{2 N-l}\left\langle\frac{Y-\frac{z+z^{\prime}}{2}}{\sqrt{\hbar}}\right\rangle^{2 M-l}\right)^{-2} \mathrm{~d} Y\right] \times \\
& \left.\qquad \iiint_{\operatorname{supp}(a)}\left|C_{l, M}(Y)\right|^{2} \mathrm{~d} Y\right] \mathrm{d} z^{\prime} .
\end{aligned}
$$

We now estimate

$$
\int_{\mathbb{R}^{2 n}}\left|C_{l, M}(Y)\right|^{2} \mathrm{~d} Y
$$

For more simplicity, we set

$$
\hat{y}:=y-\frac{q+q^{\prime}}{2} \quad \hat{\eta}:=\eta-\frac{p+p^{\prime}}{2} \quad \hat{Y}:=Y-\frac{z+z^{\prime}}{2}
$$

Noting that

$$
\left\langle\frac{\hat{Y}}{\sqrt{\hbar}}\right\rangle^{-1} \leqslant\left\langle\frac{\hat{\eta}}{\sqrt{\hbar}}\right\rangle^{-1}
$$

and that

$$
\begin{aligned}
& \sum_{|k|=0}^{2 N}\left\langle\frac{\hat{y}}{\sqrt{\hbar}}\right\rangle^{2 M}\left(1-\hbar \Delta_{u}\right)^{M} \Psi_{\hbar}^{[k]}\left(\frac{u}{\sqrt{\hbar}}, \frac{\hat{y}}{\sqrt{\hbar}}\right)= \\
& \sum_{i, j \in \hat{I}} C^{i, j}\left(\frac{\hat{y}}{\sqrt{\hbar}}\right)^{i}\left(\frac{u}{\sqrt{\hbar}}\right)^{j} \partial^{\alpha_{i, j}} \mathrm{f}_{\hbar}\left(\frac{u-\hat{y}}{\sqrt{\hbar}}\right) \overline{\partial^{\beta_{i, j}} \mathrm{f}_{\hbar}\left(\frac{\hat{y}+u}{\sqrt{\hbar}}\right)}
\end{aligned}
$$


where the $\hat{I}$ is a finite set and $C^{i, j}$ only depends on the integers $k, N, M$ and $l$, but not on the shape $\mathrm{f}_{\hbar}$, we get for $\delta>0$ small enough

$$
\begin{array}{r}
\left|C_{l, M}(Y)\right|^{2} \leqslant \sum_{i, j \in \hat{J}} \tilde{C}^{i, j}\left\langle\frac{\hat{\eta}}{\sqrt{\hbar}}\right\rangle^{-2 \tilde{M}} p_{i, j}\left((v, w) \mapsto \mathrm{f}_{\hbar}\left(\frac{v}{\sqrt{\hbar}}\right) \mathrm{f}_{\hbar}\left(\frac{w}{\sqrt{\hbar}}\right)\right)^{2 \delta} \times \\
{\left[\left|\partial^{\alpha_{i, j}} \mathrm{f}_{\hbar}\left(\frac{\cdot}{\sqrt{\hbar}}\right)\right|^{1-\delta} \star\left|\partial^{\beta_{i, j}} \mathrm{f}_{\hbar}\left(\frac{\cdot}{\sqrt{\hbar}}\right)\right|^{1-\delta}\right]^{2}}
\end{array}
$$

with again $\tilde{J}$ a finite set and $\tilde{C}^{i, j}>0$, these two only depending on the integers $k, N, M$ and $l$, but not on the shape $\mathrm{f}_{\hbar}$, and where $\star$ is the convolution product. So, integrating, we have

$$
\begin{aligned}
& \int_{\mathbb{R}^{2 n}}\left|C_{l, M}(Y)\right|^{2} \mathrm{~d} Y \leqslant \sum_{i, j \in \hat{J}} \tilde{C}^{i, j} \int_{\mathbb{R}^{n}}\left\langle\frac{\hat{\eta}}{\sqrt{\hbar}}\right\rangle^{-2 \tilde{M}} \mathrm{~d} \eta \times \\
& p_{i, j}\left(\mathrm{f}_{\hbar}\left(\frac{\cdot}{\sqrt{\hbar}}\right)\right)^{2 \delta} p_{i, j}\left(\mathrm{f}_{\hbar}\left(\frac{\cdot}{\sqrt{\hbar}}\right)\right)^{2 \delta} 2^{-n} \times \\
&\left\|\partial^{\alpha_{i, j}} \mathrm{f}_{\hbar}^{(1-\delta)}\left(\frac{\cdot}{\sqrt{\hbar}}\right)\right\|_{L^{2}\left(\mathbb{R}^{n}\right)}^{2}\left\|\partial^{\beta_{i, j}} \mathrm{f}_{\hbar}^{(1-\delta)}\left(\frac{\cdot}{\sqrt{\hbar}}\right)\right\|_{L^{1}\left(\mathbb{R}^{n}\right)}^{2}
\end{aligned}
$$

due to usual estimates on the convolution product. Noting that

$$
\left\|\partial^{\alpha_{i, j}} \mathrm{f}_{\hbar}^{(1-\delta)}\left(\frac{\cdot}{\sqrt{\hbar}}\right)\right\|_{\mathrm{L}^{2}\left(\mathbb{R}^{n}\right)}^{2} \leqslant \hbar^{-n / 2}\left\|\partial^{\alpha_{i, j}} \mathrm{f}_{\hbar}\right\|_{\mathrm{L}^{2}\left(\mathbb{R}^{n}\right)}^{2(1-2 \delta)}\left\|\partial^{\alpha_{i, j}} \mathrm{f}_{\hbar}\right\|_{\mathrm{L}^{1}\left(\mathbb{R}^{n}\right)}^{2 \delta}
$$

and that

$$
\left\|\partial^{\beta_{i, j}} \mathrm{f}_{\hbar}^{(1-\delta)}\left(\frac{\cdot}{\sqrt{\hbar}}\right)\right\|_{\mathrm{L}^{1}\left(\mathbb{R}^{n}\right)} \leqslant \hbar^{-n / 2}\left\|\partial^{\beta_{i, j}} \mathrm{f}_{\hbar}\right\|_{\mathrm{L}^{2}\left(\mathbb{R}^{n}\right)}^{(1-2 \delta)} p_{K}\left(\partial^{\beta_{i, j}} \mathrm{f}_{\hbar}\right)^{2 \delta /(1+2 \delta)} \int_{\mathbb{R}^{n}}\langle x\rangle^{K} \mathrm{~d} x
$$

for some fixed integer $K$ and for $p_{K}$ a seminorm depending on $K$, we use the following lemma to estimate the terms $\left\|\partial^{\alpha} \mathrm{f}_{\hbar}\right\|_{\mathrm{L}^{2}\left(\mathbb{R}^{n}\right)}$ and $\left\|\partial^{\beta} \mathrm{f}_{\hbar}\right\|_{\mathrm{L}^{2}\left(\mathbb{R}^{n}\right)}$. This lemma is known as the Kolmogorov inequalities.

Lemma 2.14. Let $f \in \mathscr{S}\left(\mathbb{R}^{n}\right)$. Then for any $L \in \mathbb{N}$, for any $c \in \mathbb{N}, c \leqslant L$, for any integer $i$ such that $1 \leqslant i \leqslant n$, we have

$$
\left\|\partial_{i}^{c} f\right\|_{\mathrm{L}^{2}\left(\mathbb{R}^{n}\right)} \leqslant\|f\|_{\mathrm{L}^{2}\left(\mathbb{R}^{n}\right)}^{1-\frac{c}{L}}\left\|\partial_{i}^{L} f\right\|_{\mathrm{L}^{2}\left(\mathbb{R}^{n}\right)}^{\frac{c}{L}}
$$


Then, for any $f \in \mathscr{S}\left(\mathbb{R}^{n}\right)$, for $\underline{c}=\left(c_{1}, \ldots, c_{n}\right) \in \mathbb{N}^{n}$, for $L \in \mathbb{N}, L \geqslant \max \underline{c}$, and $\underline{L}:=(L, \ldots, L)$, we have

$$
\left\|\partial^{\underline{c}} f\right\|_{\mathrm{L}^{2}\left(\mathbb{R}^{n}\right)} \leqslant\|f\|_{L^{2}\left(\mathbb{R}^{n}\right)}^{\prod_{i=1}^{n}\left(1-\frac{c_{i}}{L}\right)} \prod\left\|\partial^{L} f\right\|_{L^{2}\left(\mathbb{R}^{n}\right)}^{\prod_{i, j}\left(1-\frac{c_{i}}{L}\right) \frac{c_{j}}{L}}
$$

So,

$$
\begin{aligned}
\int_{\mathbb{R}^{2 n}}\left|C_{l, M}(Y)\right|^{2} \mathrm{~d} Y \leqslant \hbar^{-3 n / 2} C_{L, K, N, M, l}\left(\mathrm{f}_{\hbar}\right) \times & \\
& \sum_{i, j \in \hat{J}}\left\|\mathrm{f}_{\hbar}\right\|_{L^{2}}(1-2 \delta) \prod_{k}\left(1-\frac{\alpha_{i, j}^{(k)}}{L}\right)\left(1-\frac{\beta_{i, j}^{(k)}}{L}\right)
\end{aligned}
$$

for, given $i, j \in \hat{J}, \alpha_{i, j}=\left(\alpha_{i, j}^{(0)}, \ldots, \alpha_{i, j}^{(n)}\right), \beta_{i, j}=\left(\beta_{i, j}^{(0)}, \ldots, \beta_{i, j}^{(n)}\right)$, and for $L \in \mathbb{N}$, $L \geqslant \max _{i, j, k}\left(\alpha_{i, j}^{(k)}, \beta_{i, j}^{(k)}\right)$. Let now $\varepsilon>0$ : for $L$ great enough and $\delta$ small enough, from (2.11) we get

$$
\int_{\mathbb{R}^{2 n}}\left|C_{l, M}(Y)\right|^{2} \mathrm{~d} Y \leqslant \hbar^{-3 n / 2} C_{\varepsilon}\left(\mathrm{f}_{\hbar}\right)\left\|\mathrm{f}_{\hbar}\right\|_{\mathrm{L}^{2}\left(\mathbb{R}^{n}\right)}^{4-\varepsilon}
$$

with $C_{\varepsilon}\left(\mathrm{f}_{\hbar}\right)$ satisfying $(2.10)$. We then get the expected result

$$
\begin{aligned}
& \left\|\mathrm{Op}_{\hbar}^{w}(a) \varphi_{z}\right\|_{\mathrm{L}^{2}\left(\mathbb{R}^{n}\right)}^{2} \leqslant \hbar^{-3 n / 2} C_{\varepsilon}\left(\mathrm{f}_{\hbar}\right) \\
& \qquad \mathrm{f}_{\hbar} \|^{2-\varepsilon} \times \\
& \qquad \int_{\mathbb{R}^{2 n}} \iint_{\operatorname{supp}(a)}\left\langle\frac{Y-\frac{z+z^{\prime}}{2}}{\sqrt{\hbar}}\right\rangle^{-N} \mathrm{~d} Y\left\langle\frac{z-z^{\prime}}{\sqrt{\hbar}}\right\rangle^{-M} \mathrm{~d} z^{\prime}
\end{aligned}
$$

We state two applications of this result. The first one is a straightforward application of the above Proposition 2.12.

Proposition 2.15. Let $\left(\varphi_{z}\right)_{z \in \mathbb{R}^{2 n}}$ a family of coherent states with shape $\mathrm{f}_{\hbar} \in \mathfrak{s}^{[n]}$ and let $m$ be any order function on $\mathbb{R}^{2 n}$. Then, given any $\varepsilon>0$ we have the continuity property

$$
\forall a \in S(m), \forall z \in \mathbb{R}^{2 n},\left\|\mathrm{Op}_{\hbar}^{w}(a) \varphi_{z}\right\|_{\mathrm{L}^{2}\left(\mathbb{R}^{n}\right)}^{2} \leqslant \hbar^{-3 n / 2} \tilde{C}(a) C_{\varepsilon}\left(\mathrm{f}_{\hbar}\right)\left\|\mathrm{f}_{\hbar}\right\|_{\mathrm{L}^{2}\left(\mathbb{R}^{n}\right)}^{2-\varepsilon}
$$

with $\tilde{C}(a) \leqslant C \max _{0 \leqslant|\alpha| \leqslant K}\left\|m^{-1} \partial^{\alpha} a\right\|_{\mathrm{L}^{\infty}\left(\mathbb{R}^{2 n}\right)}$ where $C>0$ only depending on $m$ and with $C_{\varepsilon}\left(\mathrm{f}_{\hbar}\right)$ as in $(2.10)$.

This property gives a useful boundedness property for pseudodifferential operators acting on $\mathfrak{C}^{[n]}$. We will mainly use this property in the last part of the paper, where the shape will depend on a parameter $j \in \mathbb{N}$. The second property is about the 
localization of elements of $\mathfrak{C}^{[n]}$. We first define what mean by localization (one could also say microlocalization) of a function depending on $\hbar$.

Definition 2.16. Let $\hbar_{0}>0$ and let $\left(\varphi_{\hbar}\right)_{\hbar \in\left(0, \hbar_{0}\right]}$ be a family of Schwartz functions on $\mathbb{R}^{n}$ such that

$$
\left\|\varphi_{\hbar}\right\|_{\mathrm{L}^{2}\left(\mathbb{R}^{n}\right)}=\mathcal{O}_{\hbar}(1) .
$$

We define its semi-classical wavefront set $\mathcal{W} \mathcal{F}\left(\varphi_{\hbar}\right)$ as follows. Let $Z_{0} \in T^{*} \mathbb{R}^{n}$, then $Z_{0} \notin \mathcal{W} \mathcal{F}\left(\varphi_{\hbar}\right)$ if and only if there exists $a \in S(1)$ such that $a(Z) \geqslant \gamma>0$ for a fixed $\gamma \in \mathbb{R}$ and for $Z$ in a small neighborhood of $Z_{0}$ with

$$
\left\|\mathrm{Op}_{\hbar}^{w}(a) \varphi_{\hbar}\right\|_{\mathrm{L}^{2}\left(\mathbb{R}^{n}\right)}=\mathcal{O}\left(\hbar^{\infty}\right)
$$

Then a function $\varphi_{\hbar} \in \mathscr{S}\left(\mathbb{R}^{n}\right)$ is said localized at $X_{0} \in T^{*} \mathbb{R}^{n}$ if $\mathcal{W F}\left(\varphi_{\hbar}\right) \subset\left\{X_{0}\right\}$.

Proposition 2.17. Let $\varphi_{z}$ be a coherent state centered at $z \in T^{*} \mathbb{R}^{n}$. Let $a \in S(m)$, for some order function $m$, and assume that one can find $\delta>0$ such that a vanishes on $B(z, \delta)$, then

$$
\left\|\mathrm{Op}_{\hbar}^{w}(a) \varphi_{z}\right\|_{\mathrm{L}^{2}\left(\mathbb{R}^{n}\right)}^{2}=\tilde{C}(a) C_{\varepsilon}\left(\mathrm{f}_{\hbar}\right)\left\|\mathrm{f}_{\hbar}\right\|_{\mathrm{L}^{2}\left(\mathbb{R}^{n}\right)}^{2-\varepsilon} \mathcal{O}\left(\hbar^{\infty}\right)
$$

with $\tilde{C}(a)$ and $C_{\varepsilon}\left(\mathrm{f}_{\hbar}\right)$ as in Proposition 2.15 and with constants in $\mathcal{O}\left(\hbar^{\infty}\right)$ only depending on $\delta$.

Proof. Let $\delta>0$ and $a \in S(m)$ such that $\operatorname{supp}(a) \subset T^{*} \mathbb{R}^{n}=\mathbb{R}^{2 n} \backslash B(z, \delta)$. Then, if $\varepsilon>0$, because of Proposition 2.12, we have

$$
\begin{aligned}
& \left\|\mathrm{Op}_{\hbar}^{w}(a) \varphi_{z}\right\|_{\mathrm{L}^{2}\left(\mathbb{R}^{n}\right)}^{2} \leqslant C_{\varepsilon}\left(\mathrm{f}_{\hbar}\right)\left\|\mathrm{f}_{\hbar}\right\|_{\mathrm{L}^{2}\left(\mathbb{R}^{n}\right)}^{2-\varepsilon} \times \\
& \qquad \iint_{\mathbb{R}^{2 n}} \iint_{\mathbb{R}^{2 n} \backslash B(z, \delta)}\left\langle\frac{Y-\frac{z+z^{\prime}}{2}}{\sqrt{\hbar}}\right\rangle^{-N} \mathrm{~d} Y\left\langle\frac{z-z^{\prime}}{\sqrt{\hbar}}\right\rangle^{-M} \mathrm{~d} z^{\prime} .
\end{aligned}
$$

We denote $C_{\varepsilon}:=\tilde{C}(a) C_{\varepsilon}\left(\mathrm{f}_{\hbar}\right)\left\|\mathrm{f}_{\hbar}\right\|^{2-\varepsilon}$. Then, setting $\Upsilon:=Y-z$ and $\zeta:=z^{\prime}-z$, we get

$$
\begin{aligned}
\left\|\mathrm{Op}_{\hbar}^{w}(a) \varphi_{z}\right\|_{\mathrm{L}^{2}\left(\mathbb{R}^{n}\right)}^{2} \leqslant C_{\varepsilon} & (\underbrace{\iint_{B(0, \delta)} \iint_{\mathbb{R}^{2 n} \backslash B(0, \delta)}\left\langle\frac{\Upsilon}{\sqrt{\hbar}}\right\rangle^{-N} \mathrm{~d} \Upsilon\left\langle\frac{\zeta}{\sqrt{\hbar}}\right\rangle^{-M} \mathrm{~d} \zeta}_{(\mathbf{1})} \\
& +\underbrace{\iint_{\mathbb{R}^{2 n} \backslash B(0, \delta)} \iint_{\mathbb{R}^{2 n} \backslash B(0, \delta)}\left\langle\frac{\Upsilon}{\sqrt{\hbar}}\right\rangle^{-N} \mathrm{~d} \Upsilon\left\langle\frac{\zeta}{\sqrt{\hbar}}\right\rangle^{-M} \mathrm{~d} \zeta})
\end{aligned}
$$

and one can check that $(\mathbf{1})=\mathcal{O}\left(\hbar^{M}\right)$ and $(\mathbf{2})=\mathcal{O}\left(\hbar^{N}\right)$, so that for any integer $M, N$, we have

$$
\left\|\mathrm{Op}_{\hbar}^{w}(a) \varphi_{z}\right\|_{\mathrm{L}^{2}\left(\mathbb{R}^{n}\right)}^{2} \leqslant C_{\varepsilon} \mathcal{O}\left(\hbar^{N}+\hbar^{M}\right) .
$$


From this we get the following strong localization property.

Proposition 2.18. Let $z \in T^{*} \mathbb{R}^{n}$ and $\varphi_{z}=T_{\hbar}(z) \Lambda_{\hbar} \mathrm{f}_{\hbar} \in \mathfrak{C}^{[n]}$. Then $\varphi_{z}$ is localized at z, i.e.

$$
\mathcal{W F}\left(\varphi_{z}\right)=\{z\}
$$

Moreover if $a \in S(m)$, where $m$ is a given order function, a vanishing near $z$, then for any order function $\mu$ and any $\varepsilon>0$ we have

$$
\left\|\mathrm{Op}_{\hbar}^{w}(a) \varphi_{z}\right\|_{\mathrm{H}_{\hbar}(\mu)}=\tilde{C}(a, \mu) C_{\varepsilon}\left(\mathrm{f}_{\hbar}\right)\left\|\mathrm{f}_{\hbar}\right\|_{\mathrm{L}^{2}\left(\mathbb{R}^{n}\right)}^{1-\varepsilon} \mathcal{O}\left(\hbar^{\infty}\right)
$$

with $\tilde{C}(a, \mu), C_{\varepsilon}\left(\mathrm{f}_{\hbar}\right)$ as in Proposition 2.17.

Proof. From Proposition 2.17, we get that

$$
\mathcal{W F}\left(\varphi_{z}\right) \subset\{z\}
$$

Now, using stationary phase methods (see e.g. [32, Chapter 3]), for $a \in S(1)$ with compact support we get

$$
\left\|\mathrm{Op}_{\hbar}^{w}(a) \varphi_{z}\right\|_{\mathrm{L}^{2}\left(\mathbb{R}^{n}\right)}^{2}=|a(q, p)|^{2}\left\|\mathrm{f}_{\hbar}\right\|_{\mathrm{L}^{2}\left(\mathbb{R}^{n}\right)}^{2}+\mathcal{O}(\hbar),
$$

which gives

$$
\mathcal{W F}\left(\varphi_{z}\right)=\{z\}
$$

We now consider $a \in S(m)$ and $\gamma:=\log (\mu)$, for some order functions $m, \mu$. We have

$$
\left\|\mathrm{Op}_{\hbar}^{w}(a) \varphi_{z}\right\|_{\mathrm{H}_{\hbar}(\mu)}=\left\|e^{\gamma^{w}} \mathrm{Op}_{\hbar}^{w}(a) \varphi_{z}\right\|_{\mathrm{L}^{2}\left(\mathbb{R}^{n}\right)} .
$$

Since $L:=e^{\gamma^{w}} \mathrm{Op}_{\hbar}^{w}(a)$ is a pseudodifferential operator with its symbol in $S(m \mu)$, we may apply Proposition 2.17 to obtain the announced result.

In order to apply the above properties to the magnetic propagation of coherent states, we now introduce the magnetic normal form background.

\section{Geometry and PROpagation Under MAGNEtiC FiELD}

In this section, for the reader's convenience, we recall some recent results on the geometry and analysis of magnetic fields that will be crucial for our analsis.

The first part of this section is about the geometry underlying the magnetic field, more precisely about the classical and quantum normal forms of $\mathcal{L}_{\hbar, A}$. Classsical and quantum normal forms for the magnetic Hamiltonian were introduced by Raymond and the second author, see [25]. We first briefly present the symplectic normal form adapted to the Hamiltonian $H$, and the corresponding quantum normal form. The second part of this section recalls the long time quantum propagation estimates from [2]. 
3.1. Symplectic normal form. The following symplectic normal form result is about the existence of a symplectomorphism that locally around $\Sigma \cap \Omega$ transform the magnetic Hamiltonian $H$ into an almost integrable system.

Theorem 3.1 ([25]). Let

$$
H(q, p):=\|p-A(q)\|^{2},(q, p) \in T^{*} \mathbb{R}^{2}=\mathbb{R}^{2} \times \mathbb{R}^{2},
$$

where the magnetic potential $A: \mathbb{R}^{2} \rightarrow \mathbb{R}^{2}$ is smooth. Let $B:=\partial_{q_{1}} A_{2}-\partial_{q_{2}} A_{1}$ be the corresponding magnetic field. Let $\Omega \subset \mathbb{R}^{4}$ be a bounded open set such that $B$ does not vanish on

$$
\Omega_{0}=\left\{q \in \mathbb{R}^{2} \mid(q, A(q)) \in \Omega\right\} .
$$

Then there exists a symplectic diffeomorphism $\kappa$, defined in an open set $\tilde{\Omega} \subset \mathbb{C}_{z_{1}} \times \mathbb{R}_{z_{2}}^{2}$, with values in $T^{*} \mathbb{R}^{2}$, wich identifies the plane $\left\{z_{1}=0\right\} \cap \tilde{\Omega}$ with the surface $\{H(q, p)=$ $\left.0 \mid q \in \Omega_{0}\right\}$, and such that

$$
H \circ \kappa=\left|z_{1}\right|^{2} f\left(z_{2},\left|z_{1}\right|^{2}\right)+\mathcal{O}\left(\left|z_{1}\right|^{\infty}\right)
$$

where $f: \mathbb{R}^{2} \times \mathbb{R} \rightarrow \mathbb{R}$ is smooth. Moreover, the map

$$
\phi: \Omega_{0} \ni q \mapsto \kappa^{-1} \circ j(q) \in\left(\{0\} \times \mathbb{R}_{z_{2}}^{2}\right) \cap \tilde{\Omega}
$$

is a local diffeomorphism and

$$
f \circ(\phi(q), 0)=|B(q)|
$$

From this theorem, we get a better understanding of the dynamics near the characteristic surface $\Sigma$. Indeed, up to an $\mathcal{O}\left(\left|z_{1}\right|^{\infty}\right)$ term, that is for trajectories very close to $\Sigma$, the trajectories in the $\left(z_{1}, z_{2}\right)$ coordinates are given by the Hamiltonian $\left|z_{1}\right|^{2} f\left(z_{2},\left|z_{1}\right|^{2}\right)$ that gives the center-guide dynamics, whose center $z_{2}(t)$ follows the Hamiltonian flow led by $f\left(\cdot,\left|z_{1}\right|^{2}\right)$. Moreover, as $f\left(z_{2},\left|z_{1}\right|^{2}\right)=\tilde{B}\left(z_{2}\right)+\mathcal{O}\left(\left|z_{1}\right|^{2}\right)$, still near the surface $\Sigma$, the dynamics of the center $z_{2}(t)$ is given by the magnetic field pulled back on $\Sigma, \tilde{B}:=B \circ \phi^{-1}$.

3.2. Quantum normal form. The following theorem is the quantum version of Theorem 3.1. It introduces the normal form of $\mathcal{L}_{\hbar, A}$.

Theorem $3.2([25])$. For $\hbar$ small enough, there is a unitary Fourier integral operator $U_{\hbar}$ such that

$$
U_{\hbar}^{*} \mathcal{L}_{\hbar, A} U_{\hbar}=\mathcal{N}+R_{\hbar}
$$

where

(1) $\mathcal{N}$ is a pseudodifferential operator that commutes with

$$
\mathcal{I}_{\hbar}:=\hbar^{2} \partial_{x_{1}}^{2}+x_{1}^{2}
$$


(2) For any hermite function $h_{j}$, such that $\mathcal{I}_{\hbar} h_{j}=\hbar(2 j+1) h_{j}$, the operator $\mathcal{N}^{(j)}$ acting on $\mathrm{L}^{2}\left(\mathbb{R}_{x_{2}}\right)$ by

$$
h_{j} \otimes \mathcal{N}^{(j)}(u)=\mathcal{N}\left(h_{j} \otimes u\right)
$$

is a classical pseudodifferential operator in $S(1)$ of order 1 in $\hbar$ with its principal symbol

$$
n^{(j)}\left(x_{2}, \xi_{2}\right)=\hbar(2 j+1) B \circ \phi^{-1}\left(x_{2}, \xi_{2}\right)
$$

with $\phi$ the diffeomorphism in Theorem 3.1;

(3) Given any pseudodifferential operator $D_{\hbar}$ whose principal symbol $d_{0}$ such that $d_{0}\left(z_{1}, z_{2}\right)=c\left(z_{2}\right)\left|z_{1}\right|^{2}+\mathcal{O}\left(\left|z_{1}\right|^{3}\right)$, and any $N \geqslant 1$, there exist pseudodifferential operators $S_{\hbar, N}$ and $Z_{N}$ such that

$$
R_{\hbar}=S_{\hbar, N}\left(D_{\hbar}\right)^{N}+Z_{N},
$$

with $Z_{N}$ a pseudodifferential operator whose symbol is supported away from a fixed neighborhood of $\left|z_{1}\right|=0$.

(4) $\mathcal{N}=\mathcal{H}_{\hbar}^{0}+\mathcal{H}_{\hbar}^{1}$, where $\mathcal{H}_{\hbar}^{0}=\mathrm{Op}_{\hbar}^{w}\left(H^{0}\right), H^{0}=B \circ \phi^{-1}\left(z_{2}\right)\left|z_{1}\right|^{2}$, and the operator $\mathcal{H}_{\hbar}^{1}$ is relatively bounded with respect to $\mathcal{H}_{\hbar}^{0}$ with an arbitrarily small relative bound.

Actually, the above theorem is a slightly different formulation of the one stated in [25]. Indeed, the authors introduced a Fourier integral operator $\tilde{U}_{\hbar}$ that is unitary only microlocally in a fixed neighborhood of $\Sigma$. It will be useful for us to have a genuinely unitary operator, which microlocally satisfies the same assumptions. Since the canonical transformation associated to $U_{\hbar}$ is obtained by the flow of a compactly supported, time-dependent Hamiltonian, one can in fact obtain $U_{\hbar}$ as the quantum flow of a time-dependent, uniformly bounded self-adjoint propagator, as in Lemma 2.8; see [32, Chapter 11].

3.3. Quantum propagation under magnetic field. As is well known, for a general Schrödinger operator, and for times bigger than the Erhenfest time $\asymp|\ln \hbar|$, propagated coherent states may not remain coherent. Thus, in order to obtain a rough control on the localization of the propagated state, it is natural to estimate the growth of its Schwartz seminorms. In the case of a purely magnetic propagation, this is given by the following theorem, proved in [2]. For the purpose of this article, we only state it in the 2-dimensional case.

Theorem 3.3 ( [2]). Let $P^{t}$ be the propagator of $\mathcal{L}_{\hbar, A}$, given by Stone's theorem,

$$
P: t \in \mathbb{R} \mapsto P^{t}=e^{-\frac{i t}{\hbar} \mathcal{L}_{\hbar, A}} .
$$

Under property $(\boldsymbol{P})$, and assuming $B \geqslant b_{0}>0$, we have

$$
\forall t \in \mathbb{R}, \quad P^{t} \mathscr{S}\left(\mathbb{R}^{d}\right) \subset \mathscr{S}\left(\mathbb{R}^{d}\right) .
$$


More precisely, for all $M \in \mathbb{N}^{*}$, for any Schwartz seminorm $p$, there exist $\hbar_{0}>0$, $C>0, N \in \mathbb{N}^{*}$ and a seminorm $\tilde{p}$, such that, for all $\hbar \in\left(0, \hbar_{0}\right)$, and for all $\psi_{0} \in \mathscr{S}\left(\mathbb{R}^{d}\right)$, and all $t \in\left[0, \hbar^{-M}\right]$,

$$
p\left(P^{t} \psi_{0}\right) \leqslant C \hbar^{-N} \tilde{p}\left(\psi_{0}\right) .
$$

\section{Propagation of $\mathfrak{C}$-Class states}

Our goal is to propagate a state $\varphi_{\hbar}^{0} \in \mathfrak{C}$ through the magnetic Schrödinger equation,

$$
\left\{\begin{array}{l}
i \hbar \partial_{t} \varphi_{\hbar}^{t}=\mathcal{L}_{\hbar, A} \varphi_{\hbar}^{t} \\
\varphi_{\hbar}^{t=0}=\varphi_{\hbar}^{0},
\end{array}\right.
$$

where the initial state $\varphi_{\hbar}^{0}$ is such that

$$
\mathcal{W F}\left(\varphi_{\hbar}^{0}\right) \subset\left\{X^{0}\right\} \text { for } X^{0} \in \Sigma \cap \Omega .
$$

We consider the Fourier integral operator $U_{\hbar}$ from Theorem 3.2. Since $U_{\hbar}$ is the time-1 flow of a time-dependent quantum Hamiltonian, the same holds for the adjoint (or inverse) $U_{\hbar}^{*}$, and we may apply Proposition 2.9, which shows that $\psi_{\hbar}^{0}:=U_{\hbar}^{*} \varphi_{\hbar}^{0}$ is still a coherent state of the class $\mathfrak{C}$. Moreover, because the canonical transformation associated with $U_{\hbar}^{*}$ is the symplectomorphism $\kappa^{-1}$, we obtain that $\psi_{\hbar}^{0}$ is centered at a point $z_{0}:=\kappa^{-1}\left(X_{0}\right) \in \kappa^{-1}(\Sigma)=\left\{z_{1}=0\right\}$. Our strategy is to first propagate $\psi_{\hbar}^{0}$ through the quantum normal form $\mathcal{N}$ and then to relate it to the propagation of $\varphi_{\hbar}^{0}$ through the initial Schrödinger equation (4.1).

4.1. Propagation through the normal form. The normal form $\mathcal{N}$ of Theorem 3.2 defines an essentially self-adjoint operator on $\mathscr{S}\left(\mathbb{R}^{2}\right)$. Indeed, as

$$
\mathcal{N}=\mathcal{H}_{\hbar}^{0}+\mathcal{H}_{\hbar}^{1}
$$

with $\mathcal{H}_{\hbar}^{1}$ relatively bounded with respect to $\mathcal{H}_{\hbar}^{0}$, it suffices to prove that $\mathcal{H}_{\hbar}^{0}$ is essentially self-adjoint. This follows from the fact that $\mathcal{H}_{\hbar}^{0}$ is a tensor product of two essentially self-adjoint operators on $\mathscr{S}(\mathbb{R})$. Thus, we can study the following Cauchy problem:

$$
\left\{\begin{array}{l}
i \hbar \partial_{t} \psi_{\hbar}^{t}=\mathcal{N} \psi_{\hbar}^{t} \\
\psi_{\hbar}^{t=0}=\psi_{\hbar}^{0} .
\end{array}\right.
$$

From Stone's theorem, we have a unique family of propagators $\left(Q^{t}\right)_{t \in \mathbb{R}}$, unitary on $\mathrm{L}^{2}\left(\mathbb{R}^{2}\right)$ and satisfying

$$
\forall \psi \in \mathscr{S}\left(\mathbb{R}^{2}\right) \quad i \hbar \partial_{t} Q^{t} \psi=\mathcal{N} Q^{t} \psi
$$


Then, we denote $\psi_{\hbar}^{t}:=Q^{t} \psi_{\hbar}^{0}$ the solution to (4.2). As explained above, $\psi_{\hbar}^{0} \in \mathfrak{C}$ is localized on

$$
\kappa^{-1}\left(X^{0}\right)=\left(0, z_{2}^{0}\right)
$$

Considering the harmonic oscillator in the variable $z_{1}$,

$$
\mathcal{I}_{\hbar}=-\hbar^{2} \partial_{x_{1}}^{2}+x_{1}^{2},
$$

and the associated Hermite functions $\left(h_{j}\right)_{j \in \mathbb{N}} \subset \mathscr{S}\left(\mathbb{R}_{x_{1}}\right)$, we get the following Lemma.

Lemma 4.1. There is a family of states $\left(f_{j}^{0}\right)_{j \in \mathbb{N}} \subset \mathscr{S}\left(\mathbb{R}_{x_{2}}\right)$ and a sequence $\left(\alpha_{j}\right)_{j \in \mathbb{N}} \subset$ $\ell^{2}\left(\mathbb{R}_{+}\right)$such that

$$
\psi_{\hbar}^{0}=\sum_{j=0}^{\infty} h_{j} \otimes f_{j}^{0}
$$

with for any integer $j$, if $\alpha_{j} \neq 0$ then $\alpha_{j}^{-1} f_{j}^{0} \in \mathfrak{C}^{[1]}$, and $\mathcal{W F}^{x_{2}}\left(f_{j}^{0}\right)=\left\{z_{2}^{0}\right\}$. In the case of $\alpha_{j}=0$, we have $f_{j}=\mathcal{O}_{\mathrm{L}^{2}}\left(\hbar^{\beta}\right)$ and $\mathcal{W} \mathcal{F}^{x_{2}}\left(f_{j}^{0}\right) \subset\left\{z_{2}^{0}\right\}$.

Proof. From Definition 2.2, one can find a function $f$ in $\mathscr{S}\left(\mathbb{R}^{2}\right)$, with $\|f\|_{\mathrm{L}^{2}\left(\mathbb{R}^{2}\right)}=1$, and a family $\left(g_{\hbar}\right)_{\hbar \in\left(0, \hbar_{0}\right)} \subset \mathscr{S}\left(\mathbb{R}^{2}\right)$, with seminorms in $\mathscr{S}\left(\mathbb{R}^{2}\right)$ bounded uniformily in $\hbar$, such that

Then we let

$$
\psi_{\hbar}^{0}=T_{\hbar}\left(0, z_{2}^{0}\right) \Lambda_{\hbar}\left(f+\hbar^{\beta} g_{\hbar}\right) .
$$

$$
f_{j}^{0}:=\left\langle\psi_{\hbar}^{0}, h_{j}\right\rangle_{\mathrm{d} x_{1}}=T_{\hbar}^{\left(z_{2}\right)}\left(z_{2}^{0}\right) \Lambda_{\hbar}^{\left(z_{2}\right)}\left\langle\left(f+\hbar^{\beta} g_{\hbar}\right), h_{j}^{(\hbar=1)}\right\rangle_{\mathrm{d} x_{1}} \in \mathscr{S}\left(\mathbb{R}_{x_{2}}\right) .
$$

Since $\left(h_{j}\right)_{j \in \mathbb{N}}$ is a Hilbert basis of $\mathrm{L}^{2}\left(\mathbb{R}_{x_{1}}\right)$, after defining

$$
\forall j \in \mathbb{N}, \alpha_{j}:=\left\|\left\langle f, h_{j}^{(\hbar=1)}\right\rangle_{\mathrm{L}^{2}\left(\mathbb{R}_{x_{1}}\right)}\right\|_{\mathrm{L}^{2}\left(\mathbb{R}_{x_{2}}\right)},
$$

we get $\sum \alpha_{j}^{2}=\|f\|_{\mathrm{L}^{2}\left(\mathbb{R}^{2}\right)}^{2}=1$ and, for $j \in \mathbb{N}, \alpha_{j} \neq 0, \alpha_{j}^{-1} f_{j}^{0} \in \mathfrak{C}^{[1]}$, centered at $z_{2}^{0}$. In the case of $\alpha_{j}=0$, we get $f_{j}^{0}=\mathcal{O}_{\mathscr{S}}\left(\hbar^{\beta}\right)$ and applying Proposition 2.17 we have $\mathcal{W F}^{x_{2}}\left(f_{j}^{0}\right) \subset\left\{z_{2}^{0}\right\}$, with equality if $g_{\hbar}$ is not $\mathcal{O}_{\mathscr{S}}\left(\hbar^{\infty}\right)$ (in that case, we have $\left.\mathcal{W F}^{x_{2}}\left(f_{j}^{0}\right)=\emptyset\right)$.

Lemma 4.1 expresses $\psi_{\hbar}^{0}$ as a sum of functions, each one corresponding to a 'Landau level' of the harmonic oscillator: $f_{j}^{0}$ corresponds to the occupation of the $j^{\text {th }}$ energy level. The following lemma gives the energy distribution of these levels, in the semiclassical limit $\hbar \rightarrow 0$.

Lemma 4.2. Taking the $\hbar$-independent sequence $\left(\alpha_{j}\right)_{j \in \mathbb{N}} \subset \ell^{2}\left(\mathbb{R}_{+}\right)$introduced above, there is an $\hbar$-dependent sequence $(\varepsilon(\hbar))_{j} \subset \mathbb{R}$ such that for any $j \in \mathbb{N}$

$$
\left\|f_{j}^{0}\right\|^{2}=\alpha_{j}^{2}+\hbar^{\beta} \varepsilon_{j}(\hbar)
$$

with $\sum_{j \geqslant 0} \alpha_{j}^{2}=1$ and where $\sum_{j \geqslant 0}\left|\varepsilon_{j}(\hbar)\right|$ is bounded independently of $\hbar$. 
Proof. From the proof of Lemma 4.1 we have $\sum_{j \geqslant 0} \alpha_{j}^{2}=1$ and we can compute, using (4.3):

$$
\begin{aligned}
\left\|f_{j}^{0}\right\|_{\mathrm{L}^{2}\left(\mathbb{R}_{x_{2}}\right)}^{2}= & \left\|\left\langle f, h_{j}^{(\hbar=1)}\right\rangle_{\mathrm{d} x_{1}}\right\|_{\mathrm{d} x_{2}}^{2}+ \\
& \hbar^{\beta}\left(2 \operatorname{Re}\left(\left\langle\left\langle f, h_{j}^{(\hbar=1)}\right\rangle_{\mathrm{d} x_{1}},\left\langle g_{\hbar}, h_{j}^{(\hbar=1)}\right\rangle_{\mathrm{d} x_{1}}\right\rangle_{\mathrm{d} x_{2}}\right)+\hbar^{\beta}\left\|\left\langle g_{\hbar}, h_{j}^{(\hbar=1)}\right\rangle_{\mathrm{d} x_{1}}\right\|_{\mathrm{d} x_{2}}^{2}\right) \\
= & \alpha_{j}^{2}+\hbar^{\beta} \varepsilon_{j}(\hbar),
\end{aligned}
$$

where

$$
\left|\varepsilon_{j}(\hbar)\right| \leqslant \frac{1}{2}\left(\left\|\left\langle f, h_{j}^{(\hbar=1)}\right\rangle_{\mathrm{d} x_{1}}\right\|_{\mathrm{d} x_{2}}^{2}+\left\|\left\langle g_{\hbar}, h_{j}^{(\hbar=1)}\right\rangle_{\mathrm{d} x_{1}}\right\|_{\mathrm{d} x_{2}}^{2}\right)+\hbar^{\beta}\left\|\left\langle g_{\hbar}, h_{j}^{(\hbar=1)}\right\rangle_{\mathrm{d} x_{1}}\right\|_{\mathrm{d} x_{2}}^{2}
$$

and

$$
\sum_{j \geqslant 0}\left|\varepsilon_{j}(\hbar)\right| \leqslant\left\|g_{\hbar}\right\|_{\mathrm{L}^{2}\left(\mathbb{R}^{2}\right)}^{2}\left(\frac{1}{2}+\hbar^{\beta}\right)+1,
$$

wihch is uniformly bounded for $\hbar \in\left(0, \hbar_{0}\right)$.

Remark 4.3. The result of Lemma 4.2 can be extended to the functions $x^{\gamma} \partial_{x}^{\delta} \psi_{\hbar}^{0}$, for any $\gamma, \delta$ in $\mathbb{N}^{2}$. Indeed, we have

$$
x^{\gamma} \partial_{x}^{\delta} T_{\hbar}(z) \Lambda_{\hbar}=\hbar^{-|\delta|} T_{\hbar}(z) \Lambda_{\hbar}[q+\sqrt{\hbar} x]^{\gamma}\left[i p+\sqrt{\hbar} \partial_{x}\right]^{\delta}
$$

where we denote $z=\left(z_{1}, z_{2}\right) \in \mathbb{C} \times \mathbb{R}^{2}, z_{1}=q_{1}+i p_{1}, z_{2}=\left(q_{2}, p_{2}\right)$ and finally $q=\left(q_{1}, q_{2}\right), p=\left(p_{1}, p_{2}\right)$. Then, for $\psi_{\hbar}^{0}=T_{\hbar}(z) \Lambda_{\hbar} \cdot\left(f+\hbar^{\beta} g_{\hbar}\right)$, with $f$ and the family $\left(g_{\hbar}\right)_{\hbar}$ as in Definition 2.2, we get

$$
x^{\gamma} \partial_{x}^{\delta} \psi_{\hbar}^{0}=\hbar^{-|\delta|} T_{\hbar}(z) \Lambda_{\hbar}\left(f^{\gamma, \delta}+\hbar^{\tilde{\beta}} g_{\hbar}^{\gamma, \delta}\right),
$$

with $\tilde{\beta}=\min (1 / 2, \beta)$, where the function $f^{\gamma, \delta} \in \mathscr{S}\left(\mathbb{R}^{2}\right)$ is independent of $\hbar$, and the family $\left(g_{\hbar}^{\gamma, \delta}\right)_{\hbar}$ belongs to the Schwartz class with bounded seminorms, uniformly in $\hbar$, satisfying the definition of $\mathfrak{C}$. Then, letting

$$
x^{\gamma} \partial_{x}^{\delta} \psi_{\hbar}^{0}=\hbar^{-|\delta|} \sum_{j \in \mathbb{N}} h_{j} \otimes f_{j}^{\gamma, \delta}
$$

with $f_{j}^{\gamma, \delta}=\hbar^{|\delta|}\left\langle x^{\gamma} \partial_{x}^{\delta} \psi_{\hbar}^{0}, h_{j}\right\rangle_{\mathrm{d} x_{1}}$, mimicking the above proof, we get

$$
\left\|f_{j}^{\gamma, \delta}\right\|_{\mathrm{L}^{2}\left(\mathrm{~d} x_{2}\right)}^{2}=\left|\alpha_{j}^{\gamma, \delta}\right|^{2}+\hbar^{\tilde{\beta}} \varepsilon_{j}^{\gamma, \delta}(\hbar)
$$

where $\left(\alpha_{j}^{\gamma, \delta}\right)_{j}$ and $\left(\varepsilon_{j}^{\gamma, \delta}\right)$ are sequences as in Lemma 4.2 except that $\sum_{j}\left|\alpha_{j}^{\gamma, \delta}\right|^{2}$ is not necessarily equal to 1.

Applying Lemma 4.1, we now study the propagation of

$$
\psi_{\hbar}^{0}=\sum_{j=0}^{\infty} h_{j} \otimes f_{j}^{0} .
$$


From (3.1), the solution to (4.2) can be written as

$$
\psi_{\hbar}^{t}=\sum_{j=0}^{\infty} h_{j} \otimes f_{j}^{t}
$$

where for all $j \in \mathbb{N}, f_{j}^{t}$ satisfies the evolution equation

$$
\left\{\begin{array}{l}
i \partial_{t} f_{j}^{t}=\left(\frac{1}{\hbar} \mathcal{N}^{(j)}\right) f_{j}^{t} \\
f_{j}^{t=0}=f_{j}^{0}
\end{array}\right.
$$

where $\frac{1}{\hbar} \mathcal{N}^{(j)}$ is a semi classical pseudodifferential operator whose principal symbol is $(2 j+1) B \circ \phi^{-1}$. In order to apply usual propagation results to (4.4) we rescale time as follows. Given $j \in \mathbb{N}$, we set $\tau_{j}:=t \times \hbar(2 j+1)$ so that $\tau_{j}$ represents the reduced time corresponding to the slow drift of the $j^{\text {th }}$ landau level. So for a global time propagation $t \in\left[0, T \hbar^{-1}\right]$, we have a reduced time $\tau_{j} \in[0,(2 j+1) T]$. We let now $\tilde{f}_{j}^{\tau_{j}}:=f_{j}^{t}$ and get the following equivalent propagation equation

$$
\left\{\begin{array}{l}
i \hbar \partial_{\tau_{j}} \tilde{f}_{j}^{\tau_{j}}=\mathcal{F}^{(j)} \tilde{f}_{j}^{\tau_{j}} \\
\tilde{f}_{j}^{\tau_{j}=0}=f_{j}^{0},
\end{array}\right.
$$

where

$$
\mathcal{F}^{(j)}:=\frac{1}{\hbar(2 j+1)} \mathcal{N}^{(j)}
$$

is a pseudodifferential operator acting on $\mathrm{L}^{2}\left(\mathbb{R}_{x_{2}}\right)$ whose principal symbol is $B \circ \phi^{-1}$. At this point, we may highlight that even if the total symbol of $\mathcal{F}^{(j)}$ depends on $j$, its principal symbol does not. Since $\mathcal{N}^{(j)}$ is the restriction of an essentially selfadjoint operator to a stable subspace, it is essentially selfadjoint on $\mathrm{L}^{2}\left(\mathbb{R}_{x_{2}}\right)$. Therefore, from Stone's theorem we get a family of propagators $\left(Q_{j}^{\tau_{j}}\right)_{\tau_{j} \in \mathbb{R}}$ for this equation; coming back to the initial propagation time $t$, we obtain, for $t \in[0, T / \hbar]$,

$$
f_{j}^{t}=\tilde{f}_{j}^{\tau_{j}}=Q_{j}^{(2 j+1) \hbar t} f_{j}^{0} .
$$

Furthermore, from Lemma 4.1 and Proposition 2.9, we know that $\alpha_{j}^{-1} \tilde{f}_{j}^{\tau_{j}}$ is a coherent state in $\mathfrak{C}^{[1]}$ when $\tau_{j}$ is fixed, and the evolution of its wavefront set is governed by the Hamiltonian flow of

$$
K:=B \circ \phi^{-1},
$$

which is the pull back of the magnetic field $B$ from $\Omega_{0} \subset \mathbb{R}_{q}^{2}$ on the zero energy surface $\left\{z_{1}=0\right\}$ by $\phi=\kappa \circ\left(j_{\mid \Sigma}\right)^{-1}$. More precisely, let $\Phi_{K}^{t}$ denote the Hamiltonian flow of $K$, and let $T>0$ be such that $\Phi_{K}^{(2 j+1) \tilde{t}}\left(z_{2}^{0}\right)$ stays in the open set $\tilde{\Omega}$ for $\tilde{t} \in[0, T]$. Then, since

$$
\mathcal{W F}_{x_{2}}\left(f_{j}^{\tilde{t}=0}\right) \subset\left\{z_{2}^{0}\right\}
$$


we get

$$
\mathcal{W} \mathcal{F}_{x_{2}}\left(\tilde{f}_{j}^{\tau_{j}}\right) \subset\left\{\Phi_{K}^{\tau_{j}}\left(z_{2}^{0}\right)\right\}
$$

for $\tau_{j} \in[0,(2 j+1) T]$. In other words, for any $\tilde{t} \in[0, T]$, the wavefront set of $f_{j}^{\tilde{t} / \hbar}$ is given by

$$
\mathcal{W} \mathcal{F}_{x_{2}}\left(f_{j}^{\tilde{t} / \hbar}\right) \subset\left\{\Phi_{K}^{(2 j+1) \tilde{t}}\left(z_{2}^{0}\right)\right\}
$$

while the solution to (4.2) is given by

$$
\forall t \in[0, T / \hbar], \quad \psi_{\hbar}^{t}=\sum_{j \in \mathbb{N}} h_{j} \otimes f_{j}^{t} .
$$

We can then at this stage of the proof give an informal explanation about the quantum phenomenon occurring here. Actually, our coherent state splits into a weighted sum of coherent states, each one with a wavefront set with its own dynamics. For times $t$ of order $\hbar^{-1}$, the localization of $\psi_{\hbar}^{t}$ is no more a point in phase space but a sequence of points lying on the integral curve of the Hamiltonian provided by the magnetic field in restriction to the characteristic set, or the zero set. The point labelled by ' $j$ ' corresponds to the occupation of the $j^{\text {th }}$ Landau levels of $\mathcal{I}_{\hbar}$. Furthermore, since the Schrödinger evolution preserves the $\mathrm{L}^{2}$-norm, that is

$$
\left\|f_{j}^{t}\right\|_{\mathrm{L}^{2}\left(\mathbb{R}_{x_{2}}\right)}=\left\|f_{j}^{0}\right\|_{\mathrm{L}^{2}\left(\mathbb{R}_{x_{2}}\right)},
$$

the occupied energy level of the harmonic oscillator remains occupied with the same amount of energy, that is $\alpha_{j}^{2}$. In other words, we do not have any shift of energy to higher or lower levels during the evolution.

4.2. Back to the initial magnetic laplacian. After the study of the propagation in the normal form setting, we need to return to the initial laplacian $\mathcal{L}_{\hbar, A}$. The first step is the following lemma. Recall that from Lemma 4.1 that we write

$$
\psi_{\hbar}^{0}=\sum_{j=0}^{\infty} h_{j} \otimes f_{j}^{0} .
$$

Lemma 4.4. Let $\alpha \in(0,1)$ and for $K>0$ consider an $\hbar$ dependent integer $J_{\hbar}$ such that $J_{\hbar} \geqslant K \hbar^{-\alpha}$.

Then, for

$$
\tilde{\psi}_{\hbar}^{0}:=\sum_{j \leqslant J_{\hbar}} h_{j} \otimes f_{j}^{0},
$$

we have

$$
\psi_{\hbar}^{0}-\widetilde{\psi}_{\hbar}^{0}=\mathcal{O}_{\mathscr{S}}\left(\hbar^{\infty}\right) .
$$


Proof. Let us first recall a useful fact about Hermite coefficients of Schwartz functions. Consider some function $\psi$ in $\mathscr{S}(\mathbb{R})$. We have

$$
\psi=\sum_{j \in \mathbb{N}} c_{j} h_{j}
$$

with $c_{j}:=\left\langle\psi, h_{j}\right\rangle_{\mathrm{L}^{2}(\mathbb{R})}$. Since $\psi \in \mathscr{S}(\mathbb{R})$, for any $N \in \mathbb{N}$, for any $\hbar>0, \mathcal{I}_{\hbar}^{N} \psi \in \mathscr{S}(\mathbb{R})$. Furthermore, we have

$$
\mathcal{I}_{\hbar}^{N} \psi=\sum_{j \in \mathbb{N}} \hbar^{N}(2 j+1)^{N} c_{j} h_{j}
$$

Hence, taking the $\mathrm{L}^{2}$-norm of $\mathcal{I}_{\hbar}^{N} \psi$, and $\hbar=1$, we get

$$
\sum_{j \in \mathbb{N}}(2 j+1)^{2 N}\left|c_{j}\right|^{2}<\infty
$$

and so for any non negative integer $N$ the sequence $\left(j^{2 N}\left|c_{j}\right|^{2}\right)_{j \in \mathbb{N}}$ is bounded. Consider now the above function $\psi_{\hbar}^{0} \in \mathfrak{C} \subset \mathscr{S}\left(\mathbb{R}^{2}\right)$. We have

$$
\psi_{\hbar}^{0}=T_{\hbar}\left(0, z_{2}\right) \Lambda_{\hbar} \cdot\left(f+\hbar^{\beta} g_{\hbar}\right)
$$

with $f$ and $\left(g_{\hbar}\right)_{\hbar}$ as in Definition 2.2. In (4.7) we have $f_{j}^{0}=\left\langle\psi_{\hbar}^{0}, h_{j}\right\rangle_{\mathrm{L}^{2}\left(\mathrm{~d} x_{1}\right)}$ and $\left\|f_{j}^{0}\right\|_{\mathrm{L}^{2}\left(\mathrm{~d} x_{2}\right)}^{2}=\alpha_{j}^{2}+\hbar^{\beta} \varepsilon(\hbar)$ according to Lemma 4.2. Then, from (4.9), and by definition of the sequence $\left(\alpha_{j}\right)_{j}$, for any non negative integer $N,\left(\alpha_{j}^{2} j^{2 N}\right)_{j}$ is bounded (and independent of $\hbar$ ). Moreover, from the $\hbar$-boundedness of $\left(g_{\hbar}\right)_{\hbar}$ in $\mathscr{S}\left(\mathbb{R}^{2}\right)$, we get that for any $N,\left\|\mathcal{I}_{\hbar}^{N} g_{\hbar}\right\|_{\mathrm{L}^{2}\left(\mathbb{R}^{2}\right)}$ is bounded uniformly with $\hbar \in\left(0, \hbar_{0}\right)$. From this, we have that for any non negative integer $N,\left(j^{2 N} \varepsilon_{j}(\hbar)\right)_{j}$ is bounded uniformly with $\hbar \in\left(0, \hbar_{0}\right)$. We now turn to $(4.8)$; let

$$
\mathfrak{d}:=\psi_{\hbar}^{0}-\tilde{\psi}_{\hbar}^{0}=\sum_{j>J_{\hbar}} h_{j} \otimes f_{j}^{0}
$$

and we wish to prove that, for any $\gamma, \delta \in \mathbb{N}^{2}$

$$
\left\|x^{\gamma} \partial_{x}^{\delta} \mathfrak{d}\right\|_{\infty}=\mathcal{O}\left(\hbar^{\infty}\right)
$$

To do so, we note that

$$
x^{\gamma} \partial_{x}^{\delta} \mathfrak{d}=\sum_{j>J_{\hbar}} x_{1}^{\gamma_{1}} \partial_{x_{1}}^{\delta_{1}} h_{j} \otimes x_{2}^{\gamma_{2}} \partial_{x_{2}}^{\delta_{2}} f_{j}
$$

First, if we set

$$
f_{j}^{\gamma_{2} \delta_{2}}:=\hbar^{\delta_{2}}\left(x_{2}^{\gamma_{2}} \partial_{x_{2}}^{\delta_{2}} f_{j}\right),
$$

then because of the definition of the sequence $\left(f_{j}\right)_{j}$ and applying Remark 4.3, we get that there is a sequence of real numbers $\left(\alpha_{j}^{\gamma_{2} \delta_{2}}\right)_{j}$ independent of $\hbar$ and a family of sequences $\left(\varepsilon_{j}^{\gamma_{2} \delta_{2}}(\hbar)\right)_{j}$ uniformly bounded with respect to $\hbar \in\left(0, \hbar_{0}\right)$ such that

$$
\left\|f_{j}^{\gamma_{2} \delta_{2}}\right\|_{\mathrm{L}^{2}\left(\mathrm{~d} x_{2}\right)}^{2}=\left(\alpha_{j}^{\gamma_{2} \delta_{2}}\right)^{2}+\hbar^{\tilde{\beta}} \varepsilon_{j}^{\gamma_{2} \delta_{2}}(\hbar) .
$$


We now study the $x_{1}^{\gamma_{1}} \partial_{x_{1}}^{\delta_{1}} h_{j}$ term. One can note that from the 1-D recursion relations

$$
h_{j}^{\prime}=\sqrt{\frac{j}{2}} h_{j-1}-\sqrt{\frac{j+1}{2}} h_{j+1}
$$

and

$$
x h_{j}^{\prime}=\sqrt{\frac{j}{2}} h_{j-1}+\sqrt{\frac{j+1}{2}} h_{j+1}
$$

we get

$$
x_{1}^{\gamma_{1}} \partial_{x_{1}}^{\delta_{1}} h_{j}=\sum_{k=j-\left(\gamma_{1}+\delta_{1}\right)}^{j+\left(\gamma_{1}+\delta_{1}\right)} C_{k}^{\gamma_{1} \delta_{1} j} h_{k}
$$

where $\left(C_{k}^{\gamma_{1} \delta_{1} j}\right)_{k, j}$ is a sequence of real numbers such that for any integers $\gamma_{1}, \delta_{1}$, there exists $C_{\gamma_{1}, \delta_{1}}>0$ such that for any $k, j$ in $\mathbb{N}$,

$$
\left|C_{k}^{\gamma_{1} \delta_{1} j}\right| \leqslant C_{\gamma_{1} \delta_{1}} j^{\frac{\gamma_{1}+\delta_{1}}{2}}
$$

We finally get

$$
x^{\gamma} \partial_{x}^{\delta} \mathfrak{d}=\hbar^{-\delta_{2}} \sum_{j>J_{\hbar}}\left(\sum_{k=j-\left(\gamma_{1}+\delta_{1}\right)}^{j+\gamma_{1}+\delta_{1}} C_{k}^{\gamma_{1} \delta_{1} j} h_{k}\right) \otimes f_{j}^{\gamma_{2} \delta_{2}}
$$

that we rewrite as

$$
x^{\gamma} \partial_{x}^{\delta} \mathfrak{d}=\hbar^{-\delta_{2}} \sum_{k>J_{\hbar}-\left(\gamma_{1}+\delta_{1}\right)} h_{k} \otimes\left(\sum_{j=k-\left(\gamma_{1}+\delta_{1}\right)}^{k+\gamma_{1}+\delta_{1}} C_{k}^{\gamma_{1} \delta_{1} j} f_{j}^{\gamma_{2} \delta_{2}}\right) .
$$

Denoting

$$
\check{f}_{k}^{\gamma \delta}:=\sum_{j=k-\left(\gamma_{1}+\delta_{1}\right)}^{k+\gamma_{1}+\delta_{1}} C_{k}^{\gamma_{1} \delta_{1} j} f_{j}^{\gamma_{2} \delta_{2}}
$$

which is a Schwartz function as a finite sum of Schwartz functions on $\mathbb{R}$, we get that

$$
\left\|x^{\gamma} \partial_{x}^{\delta} \mathfrak{d}\right\|_{\mathrm{L}^{2}\left(\mathbb{R}^{2}\right)}^{2} \leqslant \hbar^{-2 \delta_{2}} \sum_{k>J_{\hbar}^{\gamma_{1} \delta_{1}}}\left\|\check{f}_{k}^{\gamma \delta}\right\|_{\mathrm{L}^{2}\left(\mathrm{~d} x_{2}\right)}^{2}
$$

with $J_{\hbar}^{\gamma_{1} \delta_{1}}:=J_{\hbar}-\left(\gamma_{1}+\delta_{1}\right)$. Note that there is a constant $\tilde{K}>0$ such that $J_{\hbar}^{\gamma_{1} \delta_{1}} \geqslant$ $\tilde{K} \hbar^{-\alpha}$. The sequence $\left((2 k+1)^{N}\left\|\check{f}_{k}^{\gamma \delta}\right\|_{\mathrm{L}^{2}\left(\mathrm{~d} x_{2}\right)}\right)_{k}$, is bounded uniformly with $\hbar \in\left(0, \hbar_{0}\right)$. 
Indeed, from above computations,

$$
\begin{aligned}
&(2 k+1)^{N}\left\|\breve{f}_{k}^{\gamma \delta}\right\|_{\mathrm{L}^{2}\left(\mathrm{~d} x_{2}\right)} \leqslant C_{\gamma_{1} \delta_{1}} \sum_{j=k-\left(\gamma_{1}+\delta_{1}\right)}^{k+\left(\gamma_{1}+\delta_{1}\right)} j^{\frac{\gamma_{1}+\delta_{1}}{2}+N}\left(\frac{2 k+1}{j}\right)^{N}\left[\left(\alpha_{j}^{\gamma_{2} \delta_{2}}\right)^{2}+\hbar_{0}^{\tilde{\beta}}\left|\varepsilon_{j}^{\gamma_{2} \delta_{2}}(\hbar)\right|\right] \\
& \leqslant \leqslant C_{N \gamma_{1} \delta_{1}} \sum_{j=k-\left(\gamma_{1}+\delta_{1}\right)}^{k+\left(\gamma_{1}+\delta_{1}\right)}\left[j^{\frac{\gamma_{1}+\delta_{1}}{2}+N}\left(\alpha_{j}^{\gamma_{2} \delta_{2}}\right)^{2}+j^{\frac{\gamma_{1}+\delta_{1}}{2}+N} \hbar_{0}^{\tilde{\beta}}\left|\varepsilon_{j}^{\gamma_{2} \delta_{2}}(\hbar)\right|\right] \\
& \leqslant\left(2\left(\gamma_{1}+\delta_{1}\right)+1\right) C_{N \gamma_{1} \delta_{1}}\left(\left\|j^{\frac{\gamma_{1}+\delta_{1}}{2}+N}\left(\alpha_{j}^{\gamma_{2} \delta_{2}}\right)^{2}\right\|_{\infty}\right. \\
&\left.+\hbar_{0}^{\tilde{\beta}}\left\|j^{\frac{\gamma_{1}+\delta_{1}}{2}+N} \varepsilon_{j}^{\gamma_{2} \delta_{2}}(\hbar)\right\|_{\infty}\right)
\end{aligned}
$$

and from what precedes, for any non negative integer $N$, the sequences $\left(\left(\alpha_{j}^{\gamma_{2} \delta_{2}}\right)^{2} j^{2 N}\right)_{j}$ and $\left(\varepsilon_{j}^{\gamma_{2} \delta_{2}}(\hbar) j^{2 N}\right)$ are bounded uniformly in $\hbar \in\left(0, \hbar_{0}\right)$. Finally, from the boundedness of $\left((2 k+1)^{N}\left\|\check{f}_{k}^{\gamma \delta}\right\|_{\mathrm{L}^{2}}\right)$, we get

$$
\begin{aligned}
\left\|x^{\gamma} \partial_{x}^{\delta} \mathfrak{d}\right\|_{\mathrm{L}^{2}\left(\mathbb{R}^{2}\right)}^{2} & =\hbar^{-2 \delta_{2}} \sum_{k>J_{\hbar}^{\gamma_{1} \delta_{1}}}\left\|\check{f}_{k}^{\gamma \delta}\right\|_{\mathrm{L}^{2}\left(\mathrm{~d} x_{2}\right)}^{2} \\
& =\hbar^{-2 \delta_{2}} \sum_{k>J_{\hbar}^{\gamma_{1} \delta_{1}}}\left(\left\|\check{f}_{k}^{\gamma \delta}\right\|_{\mathrm{L}^{2}\left(\mathrm{~d} x_{2}\right)}^{2} k^{2 N}\right)\left(k \hbar^{\alpha}\right)^{-2 N} \hbar^{2 N \alpha} \\
& \leqslant C_{\gamma \delta N} \hbar^{2\left(N \alpha-\delta_{2}\right)} \sum_{k>J_{\hbar}^{\gamma_{1} \delta_{1}}}\left(k \hbar^{\alpha}\right)^{-2 N}
\end{aligned}
$$

for any non negative integer $N$. Then, by sum-integral comparison methods, we have

$$
\left\|x^{\gamma} \partial_{x}^{\delta} \mathfrak{d}\right\|_{L^{2}\left(\mathbb{R}^{2}\right)}^{2}=\mathcal{O}\left(\hbar^{(2 N-1) \alpha-2 \delta_{2}}\right),
$$

from what we obtain that $x^{\gamma} \partial_{x}^{\delta} \mathfrak{d}=\mathcal{O}_{\mathrm{L}^{2}}\left(\hbar^{\infty}\right)$ for any multi-indices $\gamma, \delta$ in $\mathbb{N}^{2}$. So, giving any order function $m$, we get $\|\mathfrak{d}\|_{\mathrm{H}_{\hbar}(m)}=\mathcal{O}\left(\hbar^{\infty}\right)$, which is the expected result.

Remark 4.5. It appears in the above proof that for any Schwartz seminorm $p$ and for any $\delta>0$, we have

$$
\sum_{j \in \mathbb{N}} p\left(f_{j}^{0}\right)^{\delta}<\infty
$$

We will use this property later on.

Thus, this lemma teaches us that only the first Landau levels of our initial state $\psi_{\hbar}^{0}$ matter in the propagation, but we need to consider a large number of levels in 
order to get a nice estimation of the remainder. We now relate the propagation of the state

$$
\tilde{\psi}_{\hbar}^{0}:=\sum_{j \leqslant J_{\hbar}} \psi_{j, \hbar}^{0}
$$

through $\mathcal{N}$ to the propagation of $\varphi_{\hbar}^{0}$ through the initial laplacian.

Propagation under general magnetic fields. In what follows, we assume that the magnetic field $B$ does not vanish on $\Omega_{0}$, but we don't require Property $(\mathbf{P})$. Recall also from the introduction that $B$ belongs to a symbol class $S(m)$. Theorem 3.2 gives the relation between $\mathcal{L}_{\hbar, A}$ and $\mathcal{N}$,

$$
\mathcal{L}_{\hbar, A}=U_{\hbar} \mathcal{N} U_{\hbar}^{*}+U_{\hbar} R_{\hbar} U_{\hbar}^{*} .
$$

For $j \in \mathbb{N}$ and $t \in \mathbb{R}$, we let $\psi_{j, \hbar}^{t}:=h_{j} \otimes f_{j}^{t}$. From (4.4) we get the following propagation equation:

$$
i \hbar \partial_{t} U_{\hbar} \psi_{j, \hbar}^{t}=\mathcal{L}_{\hbar, A} U_{\hbar} \psi_{j, \hbar}^{t}-U_{\hbar} R_{\hbar} \psi_{j, \hbar}^{t} .
$$

Considering $\alpha \in(0,1)$ and $J_{\hbar} \in \mathbb{N}$ as in Lemma 4.4 and summing for $j \leqslant J_{\hbar}$, we get the following propagation equation for $\tilde{\psi}_{\hbar}^{t}:=\sum_{j \leqslant J_{\hbar}} h_{j} \otimes f_{j}^{t}$ :

$$
\left\{\begin{array}{l}
i \hbar \partial_{t} U_{\hbar} \tilde{\psi}_{\hbar}^{t}=\mathcal{L}_{\hbar, A} U_{\hbar} \tilde{\psi}_{\hbar}^{t}+\mathfrak{e}_{\hbar}^{t} \\
\tilde{\psi}_{\hbar}^{t=0}=\tilde{\psi}_{\hbar}^{0}
\end{array}\right.
$$

with $\mathfrak{e}_{\hbar}^{t}=U_{\hbar} R_{\hbar} \tilde{\psi}_{\hbar}^{t}$. We compare the solution to (4.10) to the solution $\varphi_{\hbar}^{t}$ to (4.1). For any $\hbar>0$ and for any $t \in \mathbb{R}$, setting $\mathcal{D}_{\hbar}^{t}=\varphi_{\hbar}-U_{\hbar} \tilde{\psi}_{\hbar}^{t}$, the Duhamel principle gives

$$
\mathcal{D}_{\hbar}^{t}=P^{t} \mathcal{D}_{\hbar}^{0}-\frac{i}{\hbar} \int_{0}^{t} P^{t-s} \mathfrak{e}_{\hbar}^{s} \mathrm{~d} s
$$

where $P^{t}$ is the propagator associated with (4.1), given by Stone's theorem. We aim at proving that for a given time $T$ and a given integer $N$, uniformly for times $t \in[0, T / \hbar]$,

$$
\left\|\mathcal{L}_{\hbar, A}{ }^{N} \mathcal{D}_{\hbar}^{t}\right\|_{L^{2}\left(\mathbb{R}^{2}\right)}=\mathcal{O}\left(\hbar^{\infty}\right)
$$

Since $P^{t}$ is unitary and commutes with $\mathcal{L}_{\hbar, A}$, we have

$$
\left\|\mathcal{L}_{\hbar, A}{ }^{N} \mathcal{D}_{\hbar}^{t}\right\|_{\mathrm{L}^{2}\left(\mathbb{R}^{2}\right)} \leqslant\left\|\mathcal{L}_{\hbar, A}{ }^{N} \mathcal{D}_{\hbar}^{0}\right\|_{L^{2}\left(\mathbb{R}^{2}\right)}+\frac{T}{\hbar^{2}} \sup _{s \in[0, T / \hbar]}\left\|\mathcal{L}_{\hbar, A}{ }^{N} \mathfrak{e}_{\hbar}^{s}\right\|_{L^{2}\left(\mathbb{R}^{2}\right)} .
$$

Since $\mathcal{L}_{\hbar, A}{ }^{N}$ is a pseudo-differential operator with symbol in $S\left(m^{N}\right)$ and $U_{\hbar}$ is unitary, we have due to Lemma 4.4

$$
\left\|\mathcal{L}_{\hbar, A}{ }^{N} \mathcal{D}_{\hbar}^{0}\right\|_{L^{2}\left(\mathbb{R}^{2}\right)} \leqslant\left\|\sum_{j>J_{\hbar}} \psi_{j, \hbar}^{0}\right\|_{\mathrm{H}_{\hbar}\left(m^{-N}\right)}=\mathcal{O}\left(\hbar^{\infty}\right) .
$$


Noting that $\left\|\mathcal{L}_{\hbar, A}{ }^{N} \mathfrak{e}_{\hbar}^{s}\right\|_{\mathrm{L}^{2}\left(\mathbb{R}^{2}\right)} \leqslant\left\|R_{\hbar} \tilde{\psi}_{\hbar}^{s}\right\|_{\mathrm{H}_{\hbar}\left(m^{-N}\right)}$, it remains to prove

$$
\sup _{s \in[0, T / \hbar]}\left\|R_{\hbar} \tilde{\psi}_{\hbar}^{s}\right\|_{\mathrm{H}_{\hbar}\left(m^{-N}\right)}=\mathcal{O}\left(\hbar^{\infty}\right) \text {. }
$$

Actually, we prove that for any order function $\mu$,

$$
\sup _{s \in[0, T / \hbar]}\left\|R_{\hbar} \tilde{\psi}_{\hbar}^{s}\right\|_{\mathrm{H}_{\hbar}(\mu)}=\mathcal{O}\left(\hbar^{\infty}\right),
$$

which implies (4.12). To do so, we begin with giving an explicit expression of $R_{\hbar}$. Using $D_{\hbar}=\mathcal{H}_{0}$ in Item 3 of Theorem 3.2, we get that for any $N \in \mathbb{N}$, there are pseudodifferential operators $S_{\hbar, N}, Z_{N}$ such that $R_{\hbar}=S_{\hbar, N} \mathcal{H}_{0}^{N}+Z_{N}$. Then, for any order function $\mu$, as $j \leqslant K \hbar^{-\alpha}$

$$
\begin{aligned}
\left\|R_{\hbar} \sum_{j \leqslant J_{\hbar}} \psi_{j, \hbar}^{t}\right\|_{\mathrm{H}_{\hbar}(\mu)} \leqslant C_{N} \hbar^{(1-\alpha) N} \sum_{j \leqslant J_{\hbar}}\left\|S_{\hbar, N} \mathrm{Op}_{\hbar}^{w}\left(B \circ \phi^{-1}\right)\left(h_{j} \otimes f_{j}^{t}\right)\right\|_{\mathrm{H}_{\hbar}(\mu)} \\
+\sum_{j \leqslant J_{\hbar}}\left\|Z_{N} \psi_{j, \hbar}^{t}\right\|_{\mathrm{H}_{\hbar}(\mu)} .
\end{aligned}
$$

From Proposition 2.15, we get that for $t \in[0, T / \hbar]$, for a given $\varepsilon \in(0,1)$,

$$
\left\|S_{\hbar, N} \mathrm{Op}_{\hbar}^{w}\left(B \circ \phi^{-1}\right)\left(h_{j} \otimes f_{j}^{t}\right)\right\|_{\mathrm{H}_{\hbar}(\mu)} \leqslant \hbar^{-3 / 2} C(\mu, N) C\left(\psi_{j, \hbar}^{t}\right)\left\|f_{j}^{0}\right\|_{\mathrm{L}^{2}\left(\mathbb{R}_{x_{2}}\right)}^{1-\varepsilon}
$$

because of $(4.6)$, with $C\left(\psi_{j, \hbar}^{t}\right)$ as in (2.12). To estimate this constant, we note that

$$
\psi_{j, \hbar}^{t}=i d_{\mathrm{L}^{2}\left(\mathbb{R}_{x_{1}}\right)} \otimes Q_{j}^{\tilde{t}} \cdot\left[h_{j} \otimes \tilde{f}_{j}^{0}\right]
$$

where $\tilde{t}=t \hbar \in[0, T]$ so from Lemma 2.8 and because of $n^{(j)} \in S(1)$, we have for any order function $\mu$

$$
\sup _{t \in[0, T / \hbar]}\left\|\psi_{j, \hbar}^{t}\right\|_{\mathrm{H}_{\hbar}(\mu)} \leqslant \sup _{\tilde{t} \in[0, T]}\left\|i d_{\mathrm{L}^{2}\left(\mathbb{R}_{x_{1}}\right)} \otimes Q_{j}^{\tilde{t}} \cdot\left[h_{j} \otimes \tilde{f}_{j}^{0}\right]\right\|_{\mathrm{H}_{\hbar}(\mu)} \leqslant C(\mu)\left\|f_{j}^{0}\right\|_{\mathrm{H}_{\hbar}(\mu)} .
$$

Then using the link between the Schwartz seminorms and the weighted Sobolev norms in Lemma 2.4, we get

$$
\sup _{t \in[0, T / \hbar]} C\left(\psi_{j, \hbar}^{t}\right) \leqslant \tilde{C}\left(f_{j}^{0}\right)
$$

and because of the definition of $f_{j}^{0},\left(\left\|f_{j}^{0}\right\|_{\mathrm{H}_{\hbar}(\nu)}\right)_{j \in \mathbb{N}}$ is a bounded sequence for any order function $\nu$, and so is $\left(\tilde{C}\left(f_{j}^{0}\right)\right)_{j \in \mathbb{N}}$. Then (4.15) becomes

$$
\sup _{t \in[0, T / \hbar]}\left(\left\|S_{\hbar, N} \mathrm{Op} \bar{\hbar}_{\hbar}^{w}\left(B \circ \phi^{-1}\right)\left(h_{j} \otimes f_{j}^{t}\right)\right\|_{\mathrm{H}_{\hbar}(\mu)}\right) \leqslant \hbar^{-3 / 2} M C(\mu, N)\left\|f_{j}^{0}\right\|_{\mathrm{L}^{2}\left(\mathbb{R}_{x_{2}}\right)}^{1-\varepsilon} .
$$

We now estimate the Schwartz seminorms of $Z_{N} \psi_{\hbar}^{t}$. Using Proposition 2.18, as $Z_{N}$ is a pseudodifferential operator with symbol $\ell_{N}$ supported away from a fixed 
neignborhood of $\left\{z_{1}=0\right\}$, where the states $\psi_{j, \hbar}^{t}$ are centered at any time $t \in[0, T / \hbar]$, and because of (4.16), we get for any order function $\mu$ and for any $\varepsilon>0$,

$$
\sup _{t \in[0, T / \hbar]}\left(\left\|Z_{N} \psi_{j, \hbar}^{t}\right\|_{\mathrm{H}_{\hbar}(\mu)}\right) \leqslant \tilde{C}\left(\ell_{N}, \mu\right) C_{\varepsilon}\left(f_{j}^{0}\right)\left\|f_{j}^{0}\right\|_{\mathrm{L}^{2}(\mathbb{R})}^{1-\varepsilon} \mathcal{O}\left(\hbar^{\infty}\right)
$$

Finally, using (4.17) and (4.18) with (4.14) we get, for any $t \in[0, T / \hbar]$,

$$
\begin{aligned}
\left\|R_{\hbar} \sum_{j \leqslant J_{\hbar}} \psi_{j, \hbar}^{t}\right\|_{\mathrm{H}_{\hbar}(\mu)} \leqslant M C_{N} C(\mu, N) \hbar^{(1-\alpha) N-3 / 2} \sum_{j \leqslant J_{\hbar}}\left\|f_{j}^{0}\right\|_{\mathrm{L}^{2}\left(\mathbb{R}^{2}\right)}^{1-\varepsilon} \\
+\tilde{C}\left(\ell_{N}, \mu\right) \mathcal{O}\left(\hbar^{\infty}\right) \sum_{j \leqslant J_{\hbar}} C_{\varepsilon}\left(f_{j}^{0}\right)\left\|f_{j}^{0}\right\|_{\mathrm{L}^{2}(\mathbb{R})}^{1-\varepsilon} .
\end{aligned}
$$

Now, from Remark 4.5, we get that

$$
\sum_{j \leqslant J_{\hbar}}\left(1+C_{\varepsilon}\left(f_{j}^{0}\right)\right)\left\|f_{j}^{0}\right\|_{\mathrm{L}^{2}(\mathbb{R})}^{1-\varepsilon}
$$

is a convergent series whose sum is bounded uniformly with $\hbar$, so that

$$
\sup _{t \in[0, T / \hbar]}\left\|R_{\hbar} \sum_{j \leqslant J_{\hbar}} \psi_{j, \hbar}^{t}\right\|_{\mathrm{H}_{\hbar}(\mu)}=\mathcal{O}\left(\hbar^{\infty}\right)
$$

for any order function $\mu$. This proves (4.13), and then we get

$$
\sup _{t \in[0, T / \hbar]}\left\|\mathcal{L}_{\hbar, A}^{N}\left(\varphi_{\hbar}^{t}-U_{\hbar} \tilde{\psi}_{\hbar}^{t}\right)\right\|_{L^{2}\left(\mathbb{R}^{2}\right)}=\mathcal{O}\left(\hbar^{\infty}\right) .
$$

Propagation under magnetic fields satisfying the property $(\mathbf{P})$. We now establish the propagation result for magnetic fields satisfying $(\mathbf{P})$. We assume henceforth that $(\mathbf{P})$ holds for $B$. We assume also there exists $b_{0}>0$ such that $\forall q \in \mathbb{R}^{2}$, $B(q) \geqslant b_{0}$. Considering again $\mathcal{D}_{\hbar}^{t}=\varphi_{\hbar}-U_{\hbar} \tilde{\psi}_{\hbar}^{t}$, from (4.11) we have

$$
\mathcal{D}_{\hbar}^{t}=P^{t} \mathcal{D}_{\hbar}^{0}-\frac{i}{\hbar} \int_{0}^{t} P^{t-s} \mathfrak{e}_{\hbar}^{s} \mathrm{~d} s
$$

and we now aim at proving that for a given $T>0$, uniformly for times $t \in[0, T / \hbar]$,

$$
\mathcal{D}_{\hbar}^{t}=\mathcal{O}_{\mathscr{S}}\left(\hbar^{\infty}\right) \text {. }
$$

We will reach this goal in two steps. We first prove that $P^{t} \mathcal{D}_{\hbar}^{0}=\mathcal{O}_{\mathscr{S}}\left(\hbar^{\infty}\right)$. We have

$$
U_{\hbar}^{*} \mathcal{D}_{\hbar}^{0}=\sum_{j>J_{\hbar}} \psi_{j, \hbar}^{0}
$$

then from Lemma 4.4, we get that

$$
U_{\hbar}^{*} \mathcal{D}_{\hbar}^{0}=\mathcal{O}_{\mathscr{S}}\left(\hbar^{\infty}\right)
$$

Applying Lemma 2.8 to $U_{\hbar}$, which is a time-1 quantum flow, we get

$$
\mathcal{D}_{\hbar}^{0}=U_{\hbar}\left(U_{\hbar}^{*} \mathcal{D}_{\hbar}^{0}\right)=\mathcal{O}_{\mathscr{S}}\left(\hbar^{\infty}\right) \text {. }
$$


Finally, applying Theorem 3.3 to $P^{t}, t \in[0, T / \hbar]$, we have the long-time estimate

$$
\sup _{t \in[0, T / \hbar]}\left(p\left(P^{t} \mathcal{D}_{\hbar}^{0}\right)\right)=\mathcal{O}\left(\hbar^{\infty}\right) .
$$

It remains now to be shown that

$$
\int_{0}^{t} P^{t-s} \mathfrak{e}_{\hbar}^{s} \mathrm{~d} s=\mathcal{O}_{\mathscr{S}}\left(\hbar^{\infty}\right) .
$$

We first prove that uniformly for times $s \in[0, T / \hbar], \mathfrak{e}_{\hbar}^{s}=\mathcal{O}_{\mathscr{S}}\left(\hbar^{\infty}\right)$. Recall that $\mathfrak{e}_{\hbar}^{t}=U_{\hbar} R_{\hbar} \sum_{j \leqslant J_{\hbar}} \psi_{j, \hbar}^{t}$ and the estimate (4.19), holding for any order function $\mu$. Using Lemma 2.4 and Lemma 2.8, we get for any Schwartz seminorm $p$,

$$
\sup _{t \in[0, T / \hbar]} p\left(U_{\hbar} R_{\hbar} \sum_{j \leqslant J_{\hbar}} \psi_{j, \hbar}^{t}\right)=\mathcal{O}\left(\hbar^{\infty}\right)
$$

and so

$$
\sup _{s \in[0, T / \hbar]} p\left(\mathfrak{e}_{\hbar}^{s}\right)=\mathcal{O}\left(\hbar^{\infty}\right)
$$

Now, applying Theorem 3.3 again, the same estimate holds for $p\left(P^{t-s} \mathfrak{e}_{\hbar}^{s}\right)$, and by the semi-norm property we may integrate and obtain (4.22). This gives

$$
\sup _{t \in[T / \hbar]} p\left(\mathcal{D}_{\hbar}^{t}\right)=\mathcal{O}\left(\hbar^{\infty}\right)
$$

In other words, letting $\varphi_{j, \hbar}^{t}=U_{\hbar} \psi_{j, \hbar}^{t}$, we have proved

$$
\sup _{t \in[0, T / \hbar]} p\left(\varphi_{\hbar}^{t}-\sum_{j \leqslant J_{\hbar}} \varphi_{j, \hbar}^{t}\right)=\mathcal{O}\left(\hbar^{\infty}\right),
$$

where $\left(\varphi_{\hbar}^{t}\right)_{t \in \mathbb{R}}$ is the solution to $(4.1)$,

4.3. End of the proof of Theorems $\mathbf{1 . 1}$ and 1.2. We consider for any $j$ the subspaces

$$
\mathcal{H}_{j, \hbar}=U_{\hbar} \cdot\left(\operatorname{span}\left(h_{j}\right) \otimes \mathrm{L}^{2}\left(\mathbb{R}_{x_{2}}\right)\right)
$$

and the operator

$$
\mathcal{J}_{\hbar}=U_{\hbar} \mathcal{I}_{\hbar} U_{\hbar}^{*}
$$

which is pseudodifferential by the Egorov theorem. Since $\left(h_{j}\right)_{j \in \mathbb{N}}$ is a Hilbert basis, the spaces $\mathcal{H}_{j, \hbar}$ are in direct sum, and

$$
\forall j \in \mathbb{N}, \forall f \in \mathcal{H}_{j, \hbar}, \mathcal{J}_{\hbar} f=(2 j+1) \hbar f .
$$


For all $t \in \mathbb{R}, \varphi_{j, \hbar}^{t}:=U_{\hbar} \psi_{j, \hbar}^{t}$, and $\psi_{j, \hbar}^{t}$ is the projection of $\psi_{\hbar}^{t}$ on the $\operatorname{space} \operatorname{span}\left(h_{j}\right) \otimes$ $\mathrm{L}^{2}\left(\mathbb{R}_{x_{2}}\right)$. Therefore, $\varphi_{j, \hbar}$ is the projection of $\varphi_{\hbar}^{t}$ on $\mathcal{H}_{j, \hbar}$, so $\varphi_{j, \hbar}^{t} \in \mathcal{H}_{j, \hbar}$, and

$$
\varphi_{\hbar}^{t}=\sum_{j \in \mathbb{N}} \varphi_{j, \hbar}^{t}
$$

This with (4.20) proves (1.4) and (1.5). Now, for each $j$, using (4.5) and Proposition 2.9 applied to the Fourier integral operator $U_{\hbar}$, we see that the wavefront set of $\varphi_{j, \hbar}^{t \hbar^{-1}}$ is located at the point

$$
\kappa\left(0, \Phi_{K}^{(2 j+1) t} z_{2}^{0}\right)=\Phi_{B}^{(2 j+1) t} X_{0}
$$

which proves (1.7). Finally, (1.6) is proved by Lemma 4.1. This ends the proof of Theorem 1.1. In order to prove Theorem 1.2, we still need to estimate the remainder $r_{\hbar}$ in Schwartz seminorms. These estimates are given by (4.23), which gives (1.9).

\section{Conclusion}

In this paper, we have shown that localized quantum particles can be split into distinct pieces by long time magnetic propagation, a property that, in similar contexts, was sometimes called quantum ubiquity. On a mathematical level, this splitting always holds modulo a small $\mathcal{O}\left(\hbar^{\infty}\right)$ term in $L^{2}$ norm (Theorem 1.1). In order to get the stronger Schwartz estimates (1.9), in our study it was necessary for the magnetic field to satisfy property $(\mathbf{P})$ and the ellipticity condition (1.8). These properties allow the use of Theorem 3.3 which is crucial in handling the remainder terms. It would be interesting to investigate whether, under magnetic confinement leading to discrete spectrum, as in [25], we could get rid of property $(\mathbf{P})$. In this case, thanks to the control on the Hamiltonian dynamics (see [25, Section 3]), one expects to get good estimates for quantum propagators up to times of order $\hbar^{-M}$ for any $M>0$. In this regime, the split wave-packets should self-interfere, giving rise to magnetic revivals (see $[30,19]$ ). We hope to return to this question in the future.

Acknowledgement : The authors are grateful to Nicolas Raymond for his important contribution to the article by bringing to their attention the property $(\mathbf{P})$, leading to the proof of Theorem 3.3.

\section{REFERENCES}

[1] J. Avron, I. Herbst, and B. Simon. Schrodinger operators with magnetic fields. i. general interactions. Duke Math. J., 45(4):847-883, 121978.

[2] G. Boil, N. Raymond, and S. Vũ Ngọc. On the stability of the schwartz class under the magnetic schrödinger flow. Preprint, 2018.

[3] C. Cheverry. Mathematical perspectives in plasma turbulence. hal-01617652, to appear in Research and Reports on Mathematics. 
[4] M. Combescure and D. Robert. Semiclassical spreading of quantum wave packets and applications near unstable fixed points of the classical flow. Asymptotic Analysis, 14, 1997.

[5] M. Combescure and D. Robert. Semiclassical spreading of quantum wave packets and applications near unstable fixed points of the classical flow. Asymptotic Analysis, 14(4):377-404, 1997.

[6] M. Combescure and D. Robert. Coherent States and Applications in Mathematical Physics. Theoretical and Mathematical Physics. Springer Netherlands, 2012.

[7] M. Dimassi and J. Sjöstrand. Spectral asymptotics in the semi-classical limit, volume 268 of London Mathematical Society Lecture Note Series. Cambridge University Press, Cambridge, 1999.

[8] A. Dufresnoy. Un exemple de champ magnetique dans $\mathbf{R}^{\nu}$. Duke Math. J., 50(3):729-734, 09 1983.

[9] G. B. Folland. Harmonic analysis in phase space. The Annals of mathematics studies 122. Princeton University Press, 1989.

[10] G. Hagedorn and A. Joye. Exponentially accurate semiclassical dynamics: Propagation, localization, ehrenfest times, scattering, and more general states. Annales Henri Poincaré, 1(5):837883, Nov 2000.

[11] G. A. Hagedorn. Semiclassical quantum mechanics. i. the $\hbar \rightarrow 0$ limit for coherent states. Comm. Math. Phys., 71(1):77-93, 1980.

[12] G. A. Hagedorn. Semiclassical quantum mechanics. iii. the large order asymptotics and more general states. Annals of Physics, 135(1):58 - 70, 1981.

[13] G. A. Hagedorn. Semiclassical quantum mechanics, iv : large order asymptotics and more general states in more than one dimension. Annales de l'I.H.P. Physique théorique, 42(4):363 $-374,1985$.

[14] G. A. Hagedorn and A. Joye. Semiclassical dynamics with exponentially small error estimates. Communications in Mathematical Physics, 207(2):439-465, Nov 1999.

[15] B. Helffer and A. Mohamed. Caractérisation du spectre essentiel de l'opérateur de schrödinger avec un champ magnétique. Annales de l'institut Fourier, 38(2):95-112, 1988.

[16] B. Helffer and A. Mohamed. Semiclassical analysis for the ground state energy of a schrodinger operator with magnetic wells. Journal of Functionnal Analysis, 138, 1996.

[17] E. J. Heller. The semiclassical way to molecular spectroscopy. Acc. Chem. Res., 14(12):368$-375,1981$.

[18] T. Ikebe and T. Kato. Uniqueness of the self-adjoint extension of singular elliptic differential operators. Arch. Rational Mech. Anal., 9:77-92, 1962.

[19] O. Lablée. Autour de la dynamique semi-classique de certains systèmes complètement intégrables. PhD thesis, Université Joseph Fourier de Grenoble 1, 2009.

[20] R. G. Littlejohn. A guiding center Hamiltonian: a new approach. J. Math. Phys., 20(12):2445$2458,1979$.

[21] T. G. Northrop. The adiabatic motion of charged particles. Interscience Tracts on Physics and Astronomy, Vol. 21. Interscience Publishers John Wiley \& Sons New York-London-Sydney, 1963.

[22] T. Paul. Semi-classical methods with emphasis on coherent states. In Quasiclassical methods (Minneapolis, MN, 1995), volume 95 of IMA Vol. Math. Appl., pages 51-88. Springer, New York, 1997.

[23] T. Paul and A. Uribe. A construction of quasi-modes using coherent states. Annales de l'I.H.P. Physique théorique, 59(4):357-381, 1993. 
[24] T. Paul and A. Uribe. On the pointwise behavior of semi-classical measures. Communications in Mathematical Physics, 175:51-88, 1996.

[25] N. Raymond and S. Vũ Ngọc. Geometry and spectrum in 2d magnetic wells. Annales de l'Institut Fourier, 2016.

[26] S. B. Reed M. Methods of mathematical physics. Functional analysis, volume Vol 1. AP, 1980.

[27] D. Robert. Autour de l'approximation semi-classique, volume 68 of Progress in Mathematics. Birkhäuser, 1987.

[28] D. Robert. Propagation of coherent states in quantum mechanics and applications. Partial Differential Equations and Applications, 15:181-252, 2007.

[29] D. Robert. La cohérence dans tout ses états. SMF - Gazette, 132, 2012.

[30] R. W. Robinett. Quantum wave packet revivals. Phys. Rep., 392(1-2):1-119, 2004.

[31] K. Yajima. Gevrey frequency set and semi-classical behaviour of wave packets. In Schrödinger operators (Aarhus, 1991), volume 403 of Lecture Notes in Phys., pages 248-264. Springer, Berlin, 1992.

[32] M. Zworski. Semiclassical Analysis. Graduate studies in mathematics. American Mathematical Society, 2012.

IRMaR (UMR 6625), University of Rennes 1, Campus de Beaulieu, 35042 Rennes CEDEX (FRANCE) 